\title{
THE SHAPIRO-LOPATINSKIJ CONDITION FOR ELLIPTIC BOUNDARY VALUE PROBLEMS
}

\author{
KATSIARYNA KRUPCHYK AND JUKKA TUOMELA
}

\begin{abstract}
Elliptic boundary value problems are well posed in suitable Sobolev spaces, if the boundary conditions satisfy the Shapiro-Lopatinskij condition. We propose here a criterion (which also covers overdetermined elliptic systems) for checking this condition. We present a constructive method for computing the compatibility operator for the given boundary value problem operator, which is also necessary when checking the criterion. In the case of two independent variables we give a formulation of the criterion for the Shapiro-Lopatinskij condition which can be checked in a finite number of steps. Our approach is based on formal theory of PDEs, and we use constructive module theory and polynomial factorisation in our test. Actual computations were carried out with computer algebra systems Singular and MuPAD.
\end{abstract}

\section{Introduction}

It is well known that elliptic boundary (value) problems are well posed only if the boundary conditions are chosen appropriately. By well-posedness one usually means that the solution exists and is unique in some space, and it depends continuously on data and parameters, or more generally that the relevant operator is at least Fredholm (the kernel and cokernel are finite-dimensional). The property which the boundary conditions should satisfy to have a well-posed problem in some Sobolev spaces for a elliptic boundary value problem is called the Shapiro-Lopatinskij condition. Of course, in many physical models the boundary conditions are more or less clear, and if the model is at all reasonable one may expect these 'natural' boundary conditions to give a well-posed problem. However, in more complicated models one may not have any natural boundary conditions, or it may not be clear which boundary conditions are 'best' in a given situation.

In [17], Mohammadi and Tuomela proposed to use the involutive form of the PDE system in numerical computations. The resulting systems are not standard ones, so the question naturally arose as to how to check effectively whether given boundary conditions indeed satisfy the Shapiro-Lopatinskij condition. The purpose of the present article is to give a (partial) answer to that question.

Originally, the Shapiro-Lopatinskij condition was formulated for square elliptic systems (that is, with as many algebraically independent equations as unknowns; see [16, 23]), but subsequently the theory was generalised to square DN-elliptic systems (elliptic in the sense of Douglis and Niremberg; see [2, 3, 4]) and overdetermined elliptic systems [8, 9]. Note that there is no need to consider overdetermined DN-elliptic systems because we proved, 
together with Seiler, in [15] that any DN-elliptic problem becomes elliptic when completed to involutive form.

The plan of the paper is as follows. In Section 2 we review the necessary background, mainly some constructive module theory and formal theory of PDEs, but we also recall some facts about Sobolev spaces. In Section 3 we prove some results related to the notion of finite type, and introduce DN-elliptic systems. Then in Section 4 we take up square DN-elliptic systems and propose a criterion for checking the Shapiro-Lopatinskij condition. We also give a constructive test for checking this condition in the case of two independent variables. This restriction is due to the fact that in the two-variable case we can use factorisation of polynomials in one variable in a way which seems to be impossible in the general case. Note that, strictly speaking, we could have treated the general case directly, but because square DN-elliptic systems are important in practice anyway, it is perhaps best to consider them separately. Also, the results of this section are helpful in proving more general results later. In Section 5 we introduce boundary value problem operators, and show how one can constructively compute their compatibility operators. These constructions are based on computing syzygies using Gröbner bases and suitable module orderings. In Section 6 we then give a new criterion for checking the Shapiro-Lopatinskij condition in the overdetermined case. We also propose a constructive test in the two-variable case. Finally, in Section 7 we draw some conclusions and indicate some directions for future work.

In our examples we have used Singular [13] and MuPAD [11]. In Appendix A we show how to use these programs to perform the computations needed.

\section{Preliminaries}

\subsection{Algebra}

All the relevant material can be found, for example, in [12] and [10] (orderings and commutative algebra) and [5] and [18] (field extensions).

\subsubsection{Orderings}

Let $\mathbb{N}_{0}^{n}$ be the space of multi indices, that is, the set of all ordered $n$-tuples $\mu=\left(\mu_{1}, \ldots, \mu_{n}\right)$ with $\mu_{i} \in \mathbb{N}_{0}$. Let $\mathbf{1}_{j}$ be a multi index whose $j$ th component is one and others are zero. The length of a multi index is $|\mu|=\mu_{1}+\ldots+\mu_{n}$ and the class of the multi-index $\mu$, denoted by cls $\mu$, is $\ell$, if $\mu_{1}=\ldots=\mu_{\ell-1}=0$ and $\mu_{\ell} \neq 0$.

A total ordering $>$ on the set of monomials $\operatorname{Mon}_{n}=\left\{\xi^{\mu} \mid \mu \in \mathbb{N}_{0}^{n}\right\}$ in $n$ variables is called a global monomial ordering if it satisfies the following conditions:

(1) $\xi^{\alpha}>\xi^{\beta}$ implies that $\xi^{\gamma} \xi^{\alpha}>\xi^{\gamma} \xi^{\beta}$ for all $\alpha, \beta, \gamma \in \mathbb{N}_{0}^{n}$, and

(2) $\xi^{\alpha}>1$ for all $\alpha \neq 0$.

In this paper we will consider only such orderings, and for simplicity we will drop the words 'global monomial' from now on. We will need the following orderings:

- lexicographic ordering (denoted by $>_{1 \mathrm{p}}$ ): $\xi^{\alpha}>_{\mathrm{lp}} \xi^{\beta}$ if and only if

$$
\exists 1 \leqslant i \leqslant n: \alpha_{1}=\beta_{1}, \ldots, \alpha_{i-1}=\beta_{i-1}, \alpha_{i}>\beta_{i} ;
$$

- degree reverse lexicographic ordering (denoted by $>_{\mathrm{dp}}$ ): $\xi^{\alpha}>_{\mathrm{dp}} \xi^{\beta}$ if and only if

$$
|\alpha|>|\beta| \text { or }\left(|\alpha|=|\beta| \text { and } \exists 1 \leqslant i \leqslant n: \alpha_{n}=\beta_{n}, \ldots, \alpha_{i+1}=\beta_{i+1}, \alpha_{i}<\beta_{i}\right) \text {; }
$$


- product ordering: let $>_{1}$ be an ordering on $\operatorname{Mon}\left(\xi_{1}, \ldots, \xi_{n}\right)$ and $>_{2}$ an ordering on $\operatorname{Mon}\left(\eta_{1}, \ldots, \eta_{l}\right)$; then the product ordering $>$ on $\operatorname{Mon}\left(\xi_{1}, \ldots, \xi_{n}, \eta_{1}, \ldots, \eta_{l}\right)$ is defined as

$$
\xi^{\alpha} \eta^{\beta}>\xi^{\alpha^{\prime}} \eta^{\beta^{\prime}} \Longleftrightarrow \xi^{\alpha}>_{1} \xi^{\alpha^{\prime}} \text { (or } \alpha=\alpha^{\prime} \text { and } \eta^{\beta}>_{2} \eta^{\beta^{\prime}} \text { ). }
$$

\subsubsection{Commutative algebra}

Let $\mathbb{A}=\mathbb{K}[\xi]=\mathbb{K}\left[\xi_{1}, \ldots, \xi_{n}\right]$ be a polynomial ring in $n$ variables where $\mathbb{K}$ is some field of characteristic zero. If $\ell$ is an ideal of $\mathbb{K}[\xi]$, then $\mathbb{A}_{\ell}=\mathbb{K}[\xi] / \ell$ is the residue class ring.

The cartesian product $\mathbb{A}^{k}$ is an $\mathbb{A}$-module of rank $k$. A module which is isomorphic to some $\mathbb{A}^{k}$ is called free. A module $M$ is finitely generated, if there are elements $a_{1}, \ldots, a_{v} \in$ $M$ such that $M=\left\langle a_{1}, \ldots, a_{\nu}\right\rangle$. Every submodule of $\mathbb{A}^{k}$ is finitely generated.

Let $C$ be a $m \times m$ matrix whose elements belong to $\mathbb{A}$. The adjoint of $C$, denoted by $\operatorname{adj}(C) \in \mathbb{A}^{m \times m}$, is the matrix of cofactors of $C$; that is,

$$
C \operatorname{adj}(C)=I_{m} \operatorname{det}(C),
$$

where $I_{m}$ is the unit matrix of size $m \times m$.

Let us consider a homomorphism $\varphi: \mathbb{A}^{k} \rightarrow \mathbb{A}^{m}$ and its image $M_{0}=$ image $(\varphi)=$ $\left\langle b^{1}, \ldots, b^{k}\right\rangle \subset \mathbb{A}^{m}$. If $s \in \mathbb{A}^{k}$ is such that

$$
s_{1} b^{1}+\ldots+s_{k} b^{k}=0,
$$

then $s$ is called a syzygy of $M_{0}$. All such $s$ form the (first) syzygy module of $M_{0}$, which is denoted by $M_{1}$. But now $M_{1}$ is the image of some homomorphism $\varphi_{1}$, and one can consider its syzygies. Now Hilbert's syzygy theorem [10, p. 45] asserts that every finitely generated $\mathbb{A}$-module has a free resolution, that is, an exact sequence of the form

$$
0 \longrightarrow \mathbb{A}^{k_{r}} \longrightarrow \mathbb{A}^{k_{r-1}} \longrightarrow \cdots \stackrel{\varphi_{2}}{\longrightarrow} \mathbb{A}^{k_{1}} \stackrel{\varphi_{1}}{\longrightarrow} \mathbb{A}^{k} \stackrel{\varphi}{\longrightarrow} \mathbb{A}^{m} \longrightarrow \mathbb{A}^{m} / M_{0} \longrightarrow 0 .
$$

Recall that 'exactness' means that the image of any map in this sequence is equal to the kernel of the subsequent map. The length of a free resolution is less than or equal to $n$, where $n$ is the number of variables in the polynomial ring $\mathbb{A}$; that is, for our module $M_{0}$ we have $r \leqslant n$.

\subsubsection{Module orderings}

Let $\mathbb{A}=\mathbb{K}[\xi]$ as before, and let us denote by $e^{i}$ the canonical basis vectors of $\mathbb{A}^{k}$. The elements of the form $\xi^{\mu} \mathrm{e}^{i}$ can be ordered in two different ways. Here, $<$ can be any monomial ordering.

- TOP ordering:

$$
\xi^{\alpha} \mathrm{e}^{i}<_{m} \xi^{\beta} \mathrm{e}^{j} \quad \text { if } \xi^{\alpha}<\xi^{\beta} \text { (or } \alpha=\beta \text { and } i>j \text { ). }
$$

- POT ordering:

$$
\xi^{\alpha} \mathrm{e}^{i}<_{m} \xi^{\beta} \mathrm{e}^{j} \quad \text { if } i>j \text { (or } i=j \text { and } \xi^{\alpha}<\xi^{\beta} \text { ). }
$$

Now let $\hat{\mathbb{A}}=\mathbb{K}[\xi, \eta]$, and let $\hat{M}$ be some submodule of $\hat{\mathbb{A}}^{k}$. We choose a TOP module ordering in $\hat{\mathbb{A}}^{k}$ and product ordering in $\hat{\mathbb{A}}$ with $\eta$ variables bigger than $\xi$. We will need the following fact [1, p. 156].

Lemma 2.1. If $\hat{G}$ is a Gröbner basis for $\hat{M}$, then $\hat{G} \cap \mathbb{A}^{k}$ is a Gröbner basis for $\hat{M} \cap \mathbb{A}^{k}$. 
Hence TOP orderings can be used to eliminate variables. On the other hand, POT orderings can be used to eliminate components. Let $M$ be a submodule of $\mathbb{A}^{k}=\mathbb{A}^{i} \oplus \mathbb{A}^{k-i}$. We choose a POT module ordering for $\mathbb{A}^{k}$, and any monomial ordering in $\mathbb{A}$. Then we have the following lemma [12, p. 177].

LEMMA 2.2. If $G$ is a Gröbner basis for $M$, then $G \cap \mathbb{A}^{k-i}$ is a Gröbner basis for $M \cap \mathbb{A}^{k-i}$.

\subsubsection{Field extensions}

An extension of a field $\mathbb{K}$ is a field $\mathbb{L}$ which contains $\mathbb{K}$ as a subfield. The extension $\mathbb{L}$ is called algebraic if every element of $\mathbb{L}$ is algebraic over $\mathbb{K}$; that is, if every element of $\mathbb{L}$ is a root of some non-zero polynomial with coefficients in $\mathbb{K}$.

If $\alpha \in \mathbb{L} \backslash \mathbb{K}$ is algebraic over $\mathbb{K}$, then there is a unique monic polynomial $p$ of least degree such that $p(\alpha)=0$. This $p$ is called a minimal polynomial of $\alpha$.

The field extension $\mathbb{L}$ is called finitely generated if there are elements $\alpha_{1}, \ldots, \alpha_{n} \in \mathbb{L} \backslash \mathbb{K}$ such that $\mathbb{L}=\mathbb{K}\left(\alpha_{1}, \ldots, \alpha_{n}\right)$. If a field extension $\mathbb{L}$ over $\mathbb{K}$ is generated by a single element $\alpha$, then $\alpha$ is called a primitive element.

The splitting field of a polynomial $p \in \mathbb{K}[x]$ is a field extension $\mathbb{L}$ of $\mathbb{K}$ over which $p$ factorizes into linear factors $x-b_{i}$ and such that the $b_{i}$ generate $\mathbb{L}$ over $\mathbb{K}$. Hence the splitting field is finitely generated and, moreover, we have the following primitive element theorem [5].

THEOREM 2.1. Let $\mathbb{K}$ be a field of characteristic 0 . Then every finitely generated extension of $\mathbb{K}$ has a primitive element. In particular, the splitting field of any polynomial $p \in \mathbb{K}[x]$ has a primitive element.

\subsection{Complex analysis}

Let $\zeta_{1}, \ldots, \zeta_{v} \in \mathbb{C}$ be some points lying in the open upper half of the complex plane. Let us define following polynomials:

$$
\begin{aligned}
& p^{+}=\left(\zeta-\zeta_{1}\right) \ldots\left(\zeta-\zeta_{\nu}\right)=\sum_{j=0}^{v} b_{j} \zeta^{j} ; \\
& p_{l}^{+}=\sum_{j=l}^{v} b_{j} \zeta^{j-l}, \quad l=1, \ldots, v .
\end{aligned}
$$

LEMMA 2.3 (see [2]). Let $\gamma_{+}$be a simple closed curve oriented counterclockwise in the upper half of the complex plane surrounding all the roots of the polynomial $p^{+}$. Then

$$
\frac{1}{2 \pi i} \oint_{\gamma_{+}} \frac{\zeta^{\tau} p_{l}^{+}(\zeta)}{p^{+}(\zeta)} d \zeta=\left\{\begin{array}{ll}
1, & \tau=l-1, \\
0, & \tau \neq l-1,
\end{array} \quad \tau=0, \ldots, v-1\right.
$$

Let $\mathfrak{M}_{+}$be the space of functions $u: \mathbb{R} \rightarrow \mathbb{C}^{m}$ which tend to zero as $x_{n} \rightarrow+\infty$ and let $v$ be a vector whose elements are in $\mathbb{C}[\zeta]$. Then we set

$$
\omega^{l}\left(x_{n}\right)=\frac{1}{2 \pi i} \oint_{\gamma_{+}} \frac{v(\zeta) p_{l}^{+}(\zeta) e^{i \zeta x_{n}}}{p^{+}(\zeta)} d \zeta, \quad l=1, \ldots, v .
$$

The following lemma is similar to some results in [14]. However, since we could not find the precise result that we needed, we give the proof below. 
Lemma 2.4. Let us suppose that $v$ is not zero modulo $p^{+}$. Then $\omega^{l} \in \mathfrak{M}_{+}, l=1, \ldots, v$, are linearly independent.

Proof. First note that $\omega^{l} \in \mathfrak{M}_{+}$, since $\mathfrak{R}(i \zeta)<0$. Let us now consider a linear combination

$$
c_{1} \omega^{1}+\ldots+c_{\nu} \omega^{\nu}=0 .
$$

Since $v$ is not divisible by $p^{+}$, we have

$$
v=q p^{+}+\mathrm{v}, \quad \mathrm{v}=\sum_{\tau=0}^{\nu-1} \mathrm{v}^{\tau} \zeta^{\tau}, \quad \mathrm{v} \neq 0,
$$

where $q, \mathrm{v}$ and $\mathrm{v}^{\tau}$ are some vectors. Let $\tau_{0}, 0 \leqslant \tau_{0}<v$, be such that

$$
\mathrm{v}^{\tau_{0}} \neq 0, \quad \mathrm{v}^{\tau}=0 \quad \text { for all } 0 \leqslant \tau<\tau_{0}
$$

So we get

$$
\omega^{l}\left(x_{n}\right)=\sum_{\tau=0}^{\nu-1} \mathrm{v}^{\tau} \frac{1}{2 \pi i} \oint_{\gamma_{+}} \frac{\zeta^{\tau} p_{l}^{+}(\zeta) e^{i \zeta x_{n}}}{p^{+}(\zeta)} d \zeta, \quad l=1, \ldots, v
$$

Lemma 2.3 implies that

$$
\frac{d^{k} \omega^{l}}{d x_{n}^{k}}(0)= \begin{cases}i^{k} v^{l-k-1}, & l>k \\ 0, & l \leqslant k\end{cases}
$$

Differentiating expression (2.3) $v-\tau_{0}-1$ times, substituting $x_{n}=0$ and using (2.5), we get

$$
\sum_{l=v-\tau_{0}}^{v} c_{l} \mathrm{v}^{l-v+\tau_{0}}=0
$$

Applying (2.4), we obtain $c_{v}=0$. Now differentiating expression (2.3) $v-\tau_{0}-2$ times, substituting $x_{n}=0$ and using (2.5) and the fact that $c_{v}=0$, we get

$$
\sum_{l=\nu-\tau_{0}-1}^{\nu-1} c_{l} \mathrm{v}^{l-\nu+\tau_{0}+1}=0 .
$$

But now (2.4) implies that $c_{\nu-1}=0$. Continuing in this way, we get $c_{\tau}=0$ for $\tau_{0}<\tau \leqslant \nu$. So (2.3) now has the following form:

$$
c_{1} \omega^{1}+\ldots+c_{\tau_{0}} \omega^{\tau_{0}}=0
$$

Now Lemma 2.3 also implies that

$$
\left.\underbrace{\int_{x_{n}}^{+\infty} \cdots \int_{x_{n}}^{+\infty}}_{k} \omega^{l}\left(x_{n}\right) d x_{n}\right|_{x_{n}=0}= \begin{cases}i^{-k} \mathrm{v}^{l+k-1}, & l \leqslant v-k, \\ 0, & l>v-k .\end{cases}
$$

Integrating (2.6) once, substituting $x_{n}=0$ and using (2.7), we get

$$
\sum_{l=1}^{\tau_{0}} c_{l} \mathbf{v}^{l}=0
$$

Hence, $c_{\tau_{0}}=0$. Continuing in this way we finally get $c_{i}=0$ for $1 \leqslant i \leqslant \tau_{0}$. 


\subsection{Geometry}

Here we simply give the basic definitions, and refer to [21] and [19] for more details. All maps and manifolds will be assumed smooth - that is, infinitely differentiable.

Let $\pi: \mathcal{E} \rightarrow \Omega$ be a bundle where $\mathcal{E}$ is called the total space, $\Omega$ the base space and $\pi$ the projection. For each $p \in \Omega$, the set $\varepsilon_{p}=\pi^{-1}(p)$ is called the fiber over $p$. All fibers are diffeomorphic to each other. A vector bundle is a bundle whose fibers are vector spaces. From now on we will assume that all bundles are vector bundles. For example, we will need the following vector bundles: the tangent bundle $T \Omega$, the cotangent bundle $T^{*} \Omega$, and the bundle of symmetric $q$-forms $S^{q}\left(T^{*} \Omega\right)$.

Given two bundles $\pi_{0}: \mathcal{E}_{0} \rightarrow \Omega_{0}$ and $\pi_{1}: \mathcal{E}_{1} \rightarrow \Omega_{1}$, the map $\Phi: \mathcal{E}_{0} \rightarrow \mathcal{E}_{1}$ is a bundle map or morphism if there is a map $\varphi$ such that the map $\left.\left.\mathcal{E}_{0}\right|_{x} \rightarrow \mathcal{E}_{1}\right|_{\varphi(x)}$ is linear, and the following diagram commutes. (Strictly speaking, the pair $(\Phi, \varphi)$ is the bundle map. However, $\varphi$ is uniquely determined by $\Phi$ (if it exists).)

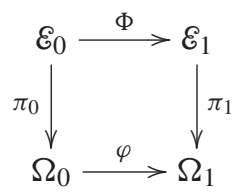

In many cases of interest we have $\Omega_{0}=\Omega_{1}$ and $\varphi=\mathrm{id}$.

Let $\pi^{q}: J_{q}(\mathcal{E}) \rightarrow \Omega$ be the bundle of $q$-jets of $\pi: \mathcal{E} \rightarrow \Omega$. If the coordinates of the base space are denoted by $\left(x_{1}, \ldots, x_{n}\right)$ and the coordinates of the fiber by $\left(y^{1}, \ldots, y^{m}\right)$, then the coordinates of $J_{q}(\mathscr{E})$ (called jet coordinates) are denoted by

$$
\left(x_{1}, \ldots, x_{n}, y^{1}, \ldots, y^{m}, \ldots, y_{\mu}^{\ell}, \ldots\right)
$$

where $|\mu| \leqslant q$. Then, denoting by $n_{q}$ the number of distinct multi indices of length $|\mu|=q$, the dimension of $J_{q}(\mathscr{E})$ is $n+m d_{q}$, where

$$
d_{q}=1+n_{1}+\ldots+n_{q}=\left(\begin{array}{c}
n+q \\
q
\end{array}\right) \quad \text { and } \quad n_{q}=\left(\begin{array}{c}
n+q-1 \\
q
\end{array}\right) .
$$

Let us also introduce the standard projections

$$
\pi_{q}^{q+r}: J_{q+r}(\mathcal{E}) \longrightarrow J_{q}(\mathcal{E})
$$

and define the embedding $\varepsilon_{q}$ by requiring that the following complex be exact:

$$
0 \longrightarrow S^{q}\left(T^{*} \Omega\right) \otimes \mathscr{E} \stackrel{\varepsilon_{q}}{\longrightarrow} J_{q}(\mathscr{E}) \stackrel{\pi_{q-1}^{q}}{\longrightarrow} J_{q-1}(\mathscr{E}) \longrightarrow 0 .
$$

Recall that a complex $\mathcal{C}$ is a sequence of bundles $\mathcal{E}_{i}$ and bundle maps $\Phi_{i}$ such that

$$
\mathcal{C}: 0 \longrightarrow \mathcal{E}_{0} \stackrel{\Phi_{0}}{\longrightarrow} \mathcal{E}_{1} \stackrel{\Phi_{1}}{\longrightarrow} \mathcal{E}_{2} \stackrel{\Phi_{2}}{\longrightarrow} \cdots
$$

and $\Phi_{i+1} \Phi_{i}=0$. A complex is exact, if $\operatorname{im}\left(\Phi_{i}\right)=\operatorname{ker}\left(\Phi_{i+1}\right)$ for all $i$.

Finally, a section of the bundle $\pi: \mathcal{E} \rightarrow \Omega$ is a map $f: \Omega \rightarrow \mathcal{E}$ such that $\pi \circ f=\mathrm{id}$. If $f$ is a section of $\mathscr{E}$, then its $q$ th prolongation, a section of $J_{q}(\mathscr{E})$, is denoted by $j^{q} f$. The vector space of smooth sections of $\mathscr{E}$ is denoted by $C^{\infty}(\mathscr{E})$, and the Sobolev spaces of sections of $\&$ by $H_{\alpha}(\mathscr{E})$. However, it is essential to consider also the case where different components of the sections are in different Sobolev spaces. Hence we consider $\alpha$ as a vector:

$$
y \in H_{\alpha}(\mathcal{E}) \Longleftrightarrow y^{i} \in H_{\alpha_{i}}\left(\mathcal{E}^{i}\right),
$$


where we have the direct sum decomposition $\mathcal{E}=\bigoplus_{i} \mathcal{E}^{i}$. The corresponding norms are then defined by

$$
\|y\|_{\alpha}=\sum_{i}\left\|y^{i}\right\|_{\alpha_{i}} .
$$

If we do not want to specify the space of sections, or if this choice is irrelevant, we will use the notation $S(\mathscr{E})$.

\section{4. $P D E$}

All the relevant materials can be found in [9], [19] and [25].

DEFinition 2.1. A (partial) differential system (or equation) of order $q$ on $\mathscr{E}$ is a subbundle $\mathcal{R}_{q}$ of $J_{q}(\mathscr{E})$. Solutions of $\mathcal{R}_{q}$ are its (local) sections.

In the present article we will consider only linear problems, so $\mathcal{R}_{q}$ will be a vector bundle.

Above, we defined what the differential equations are, but we have not yet introduced any equations. To this end, let us introduce two (vector) bundles $\varepsilon_{0}$ and $\varepsilon_{1}$, and consider the following bundle map

$$
\mathcal{A}: J_{q}\left(\mathcal{E}_{0}\right) \longrightarrow \mathcal{E}_{1} \text {. }
$$

Note that $\mathcal{A}$ is a map between finite-dimensional spaces. Then we can define a linear $q$ thorder differential operator by the formula $A=\mathcal{A} j^{q}$ :

$$
A: \mathrm{S}\left(\varepsilon_{0}\right) \longrightarrow \mathrm{S}\left(\varepsilon_{1}\right) \text {. }
$$

Now, using $\mathcal{A}$, one can represent a differential equation as a zero set of a bundle map:

$$
\mathcal{R}_{q}=\operatorname{ker}(\mathcal{A}): \mathcal{A}(x, y, \ldots)=0 .
$$

DEFINITION 2.2. The differential operator $j^{r} A: \mathrm{S}\left(\mathcal{E}_{0}\right) \rightarrow \mathrm{S}\left(J_{r}\left(\mathcal{E}_{1}\right)\right)$ is the r th prolongation of $A$. The associated morphism is denoted by $\mathcal{A}_{r}$.

Then we can define the prolongations of $\mathcal{R}_{q}$ by

$$
\mathcal{R}_{q+r}=\operatorname{ker}\left(\mathcal{A}_{r}\right) \text {. }
$$

Also, we define

$$
\mathcal{R}_{q+r}^{(s)}=\pi_{q+r}^{q+r+s}\left(\mathcal{R}_{q+r+s}\right) .
$$

Note that $\mathcal{R}_{q+r}^{(s)} \subset \mathcal{R}_{q+r}$, but in general these sets are not equal.

DEFINITION 2.3. A differential operator $A$ is called sufficiently regular if $\mathcal{R}_{q+r}^{(s)}$ is a vector bundle for all $r \geqslant 0$ and $s \geqslant 0$.

If $\Omega \subset \mathbb{R}^{n}$ and the operator $A$ has constant coefficients, then $A$ is sufficiently regular.

DEFINITION 2.4. A differential operator $A$ (of order $q$ ) is formally integrable if $A$ is sufficiently regular and $\mathcal{R}_{q+r}^{(1)}=\mathcal{R}_{q+r}$ for all $r \geqslant 0$.

The formal integrability of an operator $A$ of order $q$ means that for any $r \geqslant 1$, all the differential consequences of order $q+r$ of the relations $A y=0$ may be obtained by way of differentiations of order no more than $r$, and the application of linear algebra. 
Now it is well known that some properties of PDEs depend only on the highest-order derivative terms in the system. The information of this highest-order part is coded in the symbol of the system. In fact, there are two different kinds of symbols which are of interest here: the geometric symbol and the principal symbol.

DEFINITION 2.5. Let us consider a sufficiently regular differential equation $\mathcal{R}_{q} \subset J_{q}(\mathscr{E})$ given by $\mathcal{R}_{q}=\operatorname{ker}(\mathcal{A})$.

- The (geometric) symbol $\mathcal{M}_{q}$ of $\mathcal{R}_{q}$ is a subbundle of $S^{q}\left(T^{*} \Omega\right)$ which is defined by the commutative and exact diagram (2.10).

- The principal symbol A of operator (2.8) is a map as follows:

$$
\mathrm{A}=\mathcal{A} \varepsilon_{q}: S^{q}\left(T^{*} \Omega\right) \otimes \varepsilon_{0} \rightarrow \mathcal{E}_{1} .
$$

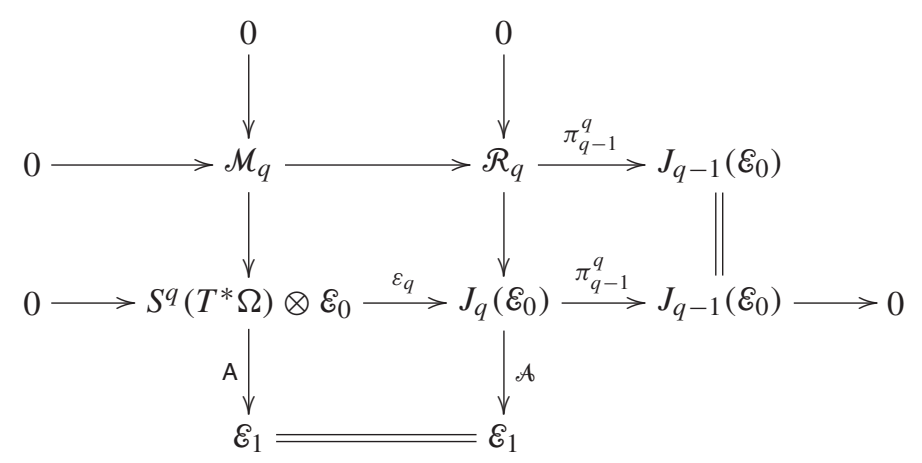

One can find more information about symbols in [19], [22] and [24].

We will now describe the symbol in a given coordinate system. Consider a linear $q$ thorder PDE given by

$$
\mathcal{R}_{q}: A y=\sum_{|\mu| \leqslant q} a_{\mu}(x) \partial^{\mu} y=f,
$$

where $x \in \Omega \subset \mathbb{R}^{n}, \Omega$ is open, $a_{\mu}(x) \in \mathbb{R}^{k \times m}$ and $\mu \in \mathbb{N}_{0}^{n}$. We will always suppose that $k \geqslant m$. Now let $M_{q}$ be the following matrix:

$$
M_{q}=\left(a_{\mu^{1}}, a_{\mu^{2}}, \ldots, a_{\mu^{n q}}\right),
$$

where $\mu^{1}>\mu^{2}>\ldots>\mu^{n_{q}}$ and $\left|\mu^{i}\right|=q$. In this way the (geometric) symbol $\mathcal{M}_{q}$ is defined by the kernel of $M_{q}$ and we may also call the matrix $M_{q}$ the symbol of $\mathcal{R}_{q}$. However, one usually considers A in a different way. Fixing some one form $\xi$ we get a bundle map $A(\xi): \varepsilon_{0} \rightarrow \mathcal{E}_{1}$, which in coordinates is given by

$$
\mathrm{A}(x, \xi)=\sum_{|\mu|=q} a_{\mu}(x) \xi^{\mu} .
$$

REMARK 2.1. We can interprete $\xi$ in four different ways:

(1) as a one form, that is, a section of $T^{*} \Omega$;

(2) as the value of this one form at a given point $p: \xi \in T_{p}^{*} \Omega$;

(3) as a vector in $\mathbb{R}^{n}$, that is, a coordinate representation of (2); and finally

(4) as indeterminates in some polynomial ring.

We think that the intended interpretation will be clear from the context. 
In terms of matrices, the connection between the two symbols is given by the formula

$$
\mathrm{A}=M_{q}\left(\Xi^{q} \otimes I\right)
$$

where $\Xi^{q}=\left(\xi^{\mu^{1}}, \xi^{\mu^{2}}, \ldots, \xi^{\mu^{n} q}\right)$. Hence, algebraically, we consider A as a module $M_{\mathrm{A}}$ generated by its rows; we may write this as $M_{\mathrm{A}} \subset \mathbb{A}^{m}$. All computations with the principal symbol are based on this interpretation.

Anyway, it turns out that the symbol contains information which helps us to recognize if the given system is formally integrable. The relevant property is called the involutivity of the symbol. However, the actual definition of involutivity is rather complicated, and because we will not need it explicitly we just refer to [24], [19], [22] and [8] for the actual definition.

The next result shows why the involutivity of the symbol is important.

THEOREM 2.2. Let us suppose that the symbol $\mathcal{M}_{q}$ is involutive, and that $\mathcal{R}_{q}$ is sufficiently regular. If no new integrability conditions are obtained by differentiating the system $\mathcal{R}_{q}$ once, then it is formally integrable.

Now we say that the system $\mathcal{R}_{q}$ is involutive if it is formally integrable and the symbol $\mathcal{M}_{q}$ is involutive.

The above discussion suggests the following algorithm to compute the involutive form of a given system.

1. The system is prolonged until its symbol becomes involutive.

2. The system is prolonged and projected once, to check whether there are integrability conditions.

3. If there are no new equations in the previous step, the system is now involutive. Otherwise go back to step 1 with the system obtained in step 2.

This is often called the Cartan-Kuranishi completion algorithm. One can show that under appropriate hypotheses the above algorithm terminates; in other words, the following theorem holds.

THEOREM 2.3. For a given sufficiently regular system $\mathcal{R}_{q}$, there are numbers $r$ and s such that $\mathcal{R}_{q+r}^{(s)}$ is involutive.

In practice, to complete a system to the involutive form we have used the DETooLs package [6] in MUPAD [11] (see also Appendix A.6 for appropriate MuPAD commands).

\subsection{Functional analysis}

We have now defined differential equations and operators, but have not yet discussed any boundary conditions. To consider boundary value problems we introduce bundles $\varepsilon_{i} \rightarrow \Omega$ where $\Omega$ is now a manifold with boundary; we denote the boundary by $\Gamma$. Further, let $g_{i} \rightarrow \Gamma$ be some bundles on the boundary. The bundle $\left.\varepsilon_{i}\right|_{\Gamma} \rightarrow \Gamma$ is the restriction of $\varepsilon_{i} \rightarrow \Omega$ to the boundary. If $f$ is a section of $\varepsilon_{i} \rightarrow \Omega$, then $\gamma f$ is the corresponding section of $\left.\varepsilon_{i}\right|_{\Gamma} \rightarrow \Gamma ; \gamma$ is called the trace map.

DEFINITION 2.6. An operator of the form

$$
\Phi: \mathrm{S}\left(\mathcal{E}_{0}\right) \times \mathrm{S}\left(g_{0}\right) \longrightarrow \mathrm{S}\left(\mathcal{E}_{1}\right) \times \mathrm{S}\left(g_{1}\right), \quad \Phi(y, z)=\left(\Phi^{11} y, \gamma \Phi^{21} y+\Phi^{22} z\right),
$$

where $\Phi^{i j}$ are differential operators, is called a differential boundary operator (DB-operator). If $g_{0}=0$, we will write

$$
\Phi(y)=(A y, B y) .
$$

In this case $\Phi$ is called a boundary value problem operator ( $B V$-operator). 
REMARK 2.2. Strictly speaking, we should write $\gamma B$ instead of $B$. However, this is the standard way of writing the BV-operator.

In terms of Sobolev spaces the most general DB-operator can be written as follows:

$$
\Phi: H_{\alpha}\left(\varepsilon_{0}\right) \times H_{\beta}\left(g_{0}\right) \longrightarrow H_{\delta}\left(\varepsilon_{1}\right) \times H_{\eta}\left(g_{1}\right)
$$

where $\alpha, \beta, \delta$ and $\eta$ are some appropriately chosen vectors.

\section{Ellipticity of the symbol}

Let us consider the system (2.11). In the geometric definition of differential equations it is natural to assume that for each $x \in \Omega \cup \Gamma \subset \mathbb{R}^{n}$ the elements of the matrices $a_{\mu}(x)$ are real. However, to formulate our problem algebraically we need to assume that the elements of $a_{\mu}$ are in some field. Since our computations are local in nature it is best to think that we fix some $x$ and then choose some convenient field. Obviously, this field should be the same for all $x$. Now for the purposes of symbolic computations we cannot take the field of real numbers; instead, we will work with the field $\mathbb{K}$, which is of characteristic zero and a subfield of real numbers. So from now on we suppose that $a_{\mu} \in \mathbb{K}^{k \times m}$ and $k \geqslant m$.

Note that although the field $\mathbb{K}$ will be the same for all $x$, various objects that we will define, such as the characteristic polynomial, will depend a priori on $x$. However, for simplicity of notation this dependence on $x$ will be suppressed.

Let $\mathrm{A}_{1}, \ldots, \mathrm{A}_{r}$ be all $m \times m$ submatrices of $\mathrm{A}$, and let us denote the corresponding minors by $p_{i}=\operatorname{det}\left(\mathrm{A}_{i}\right) \in \mathbb{K}[\xi]$. Further, let us define the (Fitting) ideal generated by these minors:

$$
\ell_{\mathrm{A}}=\left\langle p_{1}, \ldots, p_{r}\right\rangle \text {. }
$$

The complex projective variety $V_{\mathbb{C P}}\left(\ell_{A}\right)$ is called the characteristic variety of the operator A.

Definition 3.1. The differential operator $A$ is called elliptic in $\Omega$, if $A$ is injective for all real $\xi \neq 0$ and for all $x \in \Omega$.

Geometrically, this condition means that the real projective variety $V_{\mathbb{R P}}\left(\ell_{\mathrm{A}}\right)=\emptyset$.

Definition 3.2. The characteristic polynomial $p_{\mathrm{A}}$ of an elliptic operator $A$ is the greatest common divisor of its minors:

$$
p_{\mathrm{A}}=\operatorname{gcd}\left(p_{1}, \ldots, p_{r}\right)
$$

The greatest common divisor can be computed using Gröbner bases [1, p. 71]. It is immediate that $\ell_{\mathrm{A}} \subset\left\langle p_{\mathrm{A}}\right\rangle$, and it is also easy to see that the following lemma holds.

LEMMA 3.1. If $n>1$, then the characteristic polynomial does not change when the symbol is prolonged.

Proof. If A is the symbol, then $\Xi^{r} \otimes \mathrm{A}$ is the $r$-times prolonged symbol. From this the claim follows.

It is well known that $\ell_{A}$ depends only on the module generated by the rows of $A$. The same property holds also for the characteristic polynomial.

THEOREM 3.1. The characteristic polynomial $p_{\mathrm{A}}$ depends only on the module generated by the rows of $\mathrm{A}$. 
Proof. Let us denote the rows of A by $a^{i}$, and let $M=\left\langle a^{1}, \ldots, a^{k}\right\rangle$ be the module generated by these rows. Let $c \in M$, and let $\mathrm{A}_{c}$ be $\mathrm{A}$ with the added row $c$. Obviously, $p_{\mathrm{A}_{c}}$ divides $p_{\mathrm{A}}$. We will show that $p_{\mathrm{A}}$ also divides $p_{\mathrm{A}_{c}}$.

Let us denote the minors of $\mathrm{A}_{c}$ by $q_{i}$, if they are also minors of $\mathrm{A}$, and by $\tilde{q}_{i}$ otherwise. We need to show that $p_{\mathrm{A}}$ divides all $\tilde{q}_{i}$. Now $c=\sum_{i=1}^{k} p_{i} a^{i}$, where $p_{i}$ are some polynomials. Without loss of generality we can write any $\tilde{q}_{i}$ as

$$
\tilde{q}_{i}=\operatorname{det}\left(\sum_{j=1}^{k} p_{j} a^{j}, c^{1}, \ldots, c^{m-1}\right),
$$

where the $c^{i}$ are some rows of $\mathrm{A}$. But then

$$
\tilde{q}_{i}=\sum_{j=1}^{k} p_{j} \operatorname{det}\left(a^{j}, c^{1}, \ldots, c^{m-1}\right)=\sum_{j=1}^{k} p_{j} q_{j} .
$$

Hence $p_{\mathrm{A}}=p_{\mathrm{A}_{c}}$.

Now suppose that we have another basis of the module: $M=\left\langle b^{1}, \ldots, b^{s}\right\rangle$, and let us denote the corresponding matrix by $\mathrm{B}$. But all the $b^{i}$ can be represented as $b^{i}=\sum_{j=1}^{k} p_{i j} a^{j}$. By the previous argument, adding such sums to the matrix does not change the characteristic polynomial. But then, interchanging the roles of $\mathrm{A}$ and $\mathrm{B}$, we see that $p_{\mathrm{A}}=p_{\mathrm{B}}$.

\subsection{Finite type}

Definition 3.3. The symbol $\mathcal{M}_{q}$ is of finite type if $\mathcal{M}_{q+r}=0$ for some $r$.

Recall that if $\mathcal{M}_{q}$ is involutive and of finite type, then $\mathcal{M}_{q}=0$. We have the following characterisation.

THEOREM 3.2. The symbol is of finite type if and only if $\operatorname{rad}\left(\ell_{\mathrm{A}}\right)=\left\langle\xi_{1}, \ldots, \xi_{n}\right\rangle$.

Geometrically, the condition $\operatorname{rad}\left(\ell_{\mathrm{A}}\right)=\left\langle\xi_{1}, \ldots, \xi_{n}\right\rangle$ means that the characteristic variety is empty (as a projective variety).

Proof of Theorem 3.2. First suppose that $\operatorname{rad}\left(\ell_{A}\right) \neq\left\langle\xi_{1}, \ldots, \xi_{n}\right\rangle$. Hence there are $\xi \neq 0$ and $v \neq 0$ such that $\mathrm{A}(\xi) v=0$. But then by formula (2.12), $M_{q}\left(\Xi^{q} \otimes v\right)=0$. But then evidently $M_{q+r}\left(\Xi^{q+r} \otimes v\right)=0$ for all $r$, so $\mathcal{M}_{q}$ is not of finite type.

Then suppose that $\operatorname{rad}\left(\ell_{\mathrm{A}}\right)=\left\langle\xi_{1}, \ldots, \xi_{n}\right\rangle$. Let us denote by $\mathrm{e}^{i}$ the canonical basis vectors of $\mathbb{A}^{m}$, and by $M_{\mathrm{A}} \subset \mathbb{A}^{m}$ the module generated by the rows of $\mathrm{A}$. Now, operating with adjoints of submatrices of $\mathrm{A}$, it is seen that $p_{i} \mathrm{e}^{j} \in M_{\mathrm{A}}$ for all $i$ and $j$. This implies that if $f \in \ell_{\mathrm{A}}$, then $f \mathrm{e}^{j} \in M_{\mathrm{A}}$ for all $j$. Hence by hypothesis there is $s$ such that $\xi_{i}^{q+s} \mathrm{e}^{j} \in M_{\mathrm{A}}$ for all $i$ and $j$. But then there must be some $r$ such that $\xi_{i}^{\mu} \mathrm{e}^{j} \in M_{\mathrm{A}}$ for all $|\mu|=q+r$ and all $j$. Hence the symbol $\mathcal{M}_{q+r}$ has a representation such that the symbol matrix $M_{q+r}$ contains an identity matrix with $n_{q+r} m$ columns. Hence $\operatorname{ker}\left(M_{q+r}\right)=0$.

Corollary 3.1. If $n>1$ and $\mathcal{M}_{q}$ is of finite type, then $p_{\mathrm{A}}=1$.

Proof. Since $\ell_{\mathrm{A}} \subset\left\langle p_{\mathrm{A}}\right\rangle$, we have $V_{\mathbb{C P}}\left(\left\langle p_{\mathrm{A}}\right\rangle\right) \subset V_{\mathbb{C P}}\left(\ell_{\mathrm{A}}\right)$. But if $p_{\mathrm{A}} \neq 1$, then $V_{\mathbb{C P}}\left(\left\langle p_{\mathrm{A}}\right\rangle\right)$ is not empty.

In general, the converse of the above result is not true. For example if $p_{1}=\xi_{1}^{2}+\xi_{2}^{2}+\xi_{3}^{2}$ and $p_{2}=2 \xi_{1}^{2}+\xi_{2}^{2}+\xi_{3}^{2}$, then

$$
\operatorname{gcd}\left(p_{1}, p_{2}\right)=1 \quad \text { but } \quad \operatorname{rad}\left(\left\langle p_{1}, p_{2}\right\rangle\right)=\left\langle\xi_{1}, \xi_{2}^{2}+\xi_{3}^{2}\right\rangle .
$$


However, we have the following partial converse.

Lemma 3.2. In the two-dimensional case, $p_{\mathrm{A}}=1$ implies that $\mathcal{M}_{q}$ is of finite type.

Proof. Let $f_{i}\left(\xi_{1}\right)=p_{i}\left(\xi_{1}, 1\right)$ and $g_{i}\left(\xi_{2}\right)=p_{i}\left(1, \xi_{2}\right)$. Now if $p_{\mathrm{A}}=1$ we have also

$$
\operatorname{gcd}\left(f_{1}, \ldots, f_{r}\right)=1 \quad \text { and } \quad \operatorname{gcd}\left(g_{1}, \ldots, g_{r}\right)=1 .
$$

But this implies that there are polynomials $a_{i} \in \mathbb{K}\left[\xi_{1}\right]$ and $b_{i} \in \mathbb{K}\left[\xi_{2}\right]$ such that

$$
\sum_{i=1}^{r} a_{i} f_{i}=1 \quad \text { and } \quad \sum_{i=1}^{r} b_{i} g_{i}=1 .
$$

Homogenizing these equations shows that there are numbers $s_{i}$ such that $\xi_{i}^{s_{i}} \in \ell_{\mathrm{A}}$. Hence $\operatorname{rad}\left(\ell_{\mathrm{A}}\right)=\left\langle\xi_{1}, \xi_{2}\right\rangle$.

One may interpret the above results geometrically as follows. In a typical situation, our problem is given in some domain $\Omega \subset \mathbb{R}^{n}$, and the boundary $\Gamma$ is an $(n-1)$ dimensional submanifold of the closure of $\Omega$. Hence the codimension of $\Gamma$ is one. Now the fact that $p_{\mathrm{A}}=1$ implies that one cannot impose any conditions in a set $S \subset \Omega \cup \Gamma$ of codimension one. However, it may be possible to give some conditions, if $\operatorname{codim}(S)>1$. But if $n=2$ and $\operatorname{codim}(S)>1$, then $\operatorname{dim}(S)=0$; that is, the system is of finite type. On the other hand, if $n>2$, then the 'intermediate' situations $\operatorname{codim}(S)>1$ and $\operatorname{dim}(S)>0$ can really occur. In [4] and [20] one can find some results for these kinds of problems.

\subsection{Weights}

To generalise the notion of ellipticity, Douglis and Nirenberg [7] introduced the concept of weights of the system. In [15] it is shown that any system that is elliptic with respect to the generalised definition becomes elliptic when completed to the involutive form. So the apparent generality of ellipticity is just the result of restricting our attention to square systems. Hence this concept is interesting only in the square case, so when discussing DNelliptic systems (elliptic in the sense of Douglis-Nirenberg) we will always suppose that $k=m$.

The weights are two sets of integers: we denote by $s_{l}$ the weights for the equations, $1 \leqslant l \leqslant m$, and by $t_{j}$ the weights for the unknowns, $1 \leqslant j \leqslant m$. They must be chosen such that

$$
s_{l}+t_{j} \geqslant q_{l j}
$$

where $q_{l j}$ is the maximal order of a derivative of the $j$ th unknown function in the $l$ th equation and if $s_{l}+t_{j}<0$ then there are no derivatives of the $j$ th unknown function in the $l$ th equation of the system.

Definition 3.4. The weighted (principal) symbol of the differential operator $A$ is

$$
\left(\mathrm{A}_{w}\right)_{l, j}=\sum_{|\mu|=s_{l}+t_{j}}\left(a_{\mu}(x)\right)_{l, j} \xi^{\mu} .
$$

The operator $A$ is $D N$-elliptic if $\mathrm{A}_{w}$ is injective for all real $\xi \neq 0$ and for all $x \in \Omega$. (Note that in all cases we mean the submatrix of $A$, except for the notation $A_{w}$, which we use for the weighted principal symbol.) 
In particular, ordinary ellipticity is a special case of DN-ellipticity with weights

$$
s_{1}=\ldots=s_{m}=0 \quad \text { and } \quad t_{1}=\ldots=t_{m}=q .
$$

Note that without loss of generality one may suppose, if convenient, that $s_{1} \leqslant s_{2} \leqslant \ldots \leqslant$ $s_{m}=0$ and $t_{1} \geqslant t_{2} \geqslant \ldots \geqslant t_{m} \geqslant 1$. It is not difficult to see that DN-ellipticity is not invariant with respect to changing the dependent variables.

The following is a natural analog of Definition 3.2.

DefinITION 3.5. The characteristic polynomial of a DN-elliptic operator $A$ is

$$
p_{\mathrm{A}}=\operatorname{det}\left(\mathrm{A}_{w}\right) .
$$

This is a homogeneous polynomial in $\mathbb{K}[\xi]$ of degree $\sum_{l=1}^{m}\left(s_{l}+t_{l}\right)$.

Now, whether the characteristic polynomial is defined by Definition 3.2 or by Definition 3.5 , we will need the following property. Let us set $\xi=\left(\xi^{\prime}, \zeta\right)$, so when we fix some $\xi^{\prime}$ we may view $p_{\mathrm{A}}$ as a polynomial of a single variable $\zeta$. Since $\xi^{\prime} \in \mathbb{R}^{n-1}$, we have $p_{\mathrm{A}} \in \mathbb{R}[\zeta]$ in this interpretation, and $p_{\mathrm{A}}$ will be different for each different $\xi^{\prime}$. However, we will still use the symbol $p_{\mathrm{A}}$; we hope that the intended meaning will be clear from the context. A similar convention will be used later on with other objects.

DEFINITION 3.6. The operator $A$ is properly elliptic if it is elliptic and at all boundary points and for all $\xi^{\prime} \neq 0$ its characteristic polynomial $p_{\mathrm{A}} \in \mathbb{R}[\zeta]$ has equally many roots in the upper and lower half of the complex plane. Likewise, the operator $A$ is properly $D N$-elliptic if it is DN-elliptic and at all boundary points and for all $\xi^{\prime} \neq 0$ its characteristic polynomial $p_{\mathrm{A}} \in \mathbb{R}[\zeta]$ has equally many roots in the upper and lower half of the complex plane.

Hence, for a properly (DN-)elliptic operator $A$, the degree of the characteristic polynomial $p_{\mathrm{A}}$ must be even. From now on we will always suppose that $p_{\mathrm{A}} \neq 1$, and we denote the degree of the characteristic polynomial by $2 v>0$.

Note that for $n>2$, proper (DN-)ellipticity follows from (DN-)ellipticity. If the coefficients of the characteristic polynomial are real functions, then proper ellipticity also follows from ellipticity in the case $n=2$. In this paper we will only consider systems with real coefficients, so for our purposes ellipticity and proper ellipticity are equivalent. Hence we will drop the word 'proper' from now on.

Consider now a boundary operator

$$
B y=g, \quad x \in \Gamma,
$$

where $B$ is of size $\tilde{v} \times m$. For the purposes of this paper we may suppose that $\Gamma$ is smooth. To define the weighted principal symbol of the boundary operator, we introduce a third set of integers $r_{1}, \ldots, r_{v}$ such that

$$
r_{l}+t_{j} \geqslant q_{l j}^{b}
$$

where $q_{l j}^{b}$ is the maximal order of a derivative of the $j$ th unknown function in the $l$ th boundary condition, and if $r_{l}+t_{j}<0$ then there are no derivatives of the $j$ th unknown function in the $l$ th boundary condition.

Then the weighted (principal) symbol of the boundary operator $B$ is

$$
\left(\mathrm{B}_{w}\right)_{l, j}=\sum_{|\mu|=r_{l}+t_{j}}\left(b_{\mu}(x)\right)_{l, j} \xi^{\mu} .
$$


We will suppose that for any fixed $x$ the elements of $b_{\mu}$ are in the same field $\mathbb{K}$ as the elements of $a_{\mu}$. Note that when we pass to the involutive form we do not need any weights for the operator itself. However, we still need weights for the boundary operator.

REMARK 3.1. Usually, in the definition of (weighted) symbols one replaces the derivative $\partial^{\mu}$ in the original operator by $i^{|\mu|} \xi^{\mu}$. However, for simplicity of notation we delete the imaginary factor because it turns out that

(i) it is not needed in the definition of ellipticity because the characteristic polynomial is homogeneous;

(ii) it is not necessary in the criteria for the Shapiro-Lopatinskij condition either. We will explain this in an appropriate place.

\section{Square DN-elliptic boundary problems}

In this section we will treat the square DN-elliptic case, and will consider the overdetermined case later. We will assume that the boundary operator has $v$ rows, where $2 v$ is the degree of the characteristic polynomial. The reason for choosing $v$ conditions is that if there were fewer boundary conditions the problem would be underdetermined, and if there were more conditions the boundary conditions should satisfy some compatibility conditions. The latter case is a special case of the more general case considered in Section 6.

The relevant condition which guarantees the well-posedness of the boundary problem is called the Shapiro-Lopatinskij condition or the complementing condition; from now on we will abbreviate this as the SL-condition.

Let $\Omega$ be a smooth manifold with boundary, and let $\Gamma$ be its boundary. Let us fix some $p \in \Gamma$. Then there are some neighborhood $U$ of $p$ and local coordinates on $U$ such that $U \cap \Omega$ is given in these coordinates by the half space $x_{n} \geqslant 0$, and $U \cap \Gamma$ is given by $x_{n}=0$. From now on we will always work with these local coordinates, and will write $x=\left(x^{\prime}, x_{n}\right)$ where $x^{\prime}=\left(x_{1}, \ldots, x_{n-1}\right)$.

Now, fixing some boundary point $\left(x^{\prime}, 0\right) \in \Gamma$ and some $\xi^{\prime} \neq 0$, we consider the following ordinary differential operators with constant coefficients:

$$
\left\{\begin{array}{l}
\mathrm{A}_{w}\left(x^{\prime}, 0, \xi^{\prime}, D_{n}\right) u\left(x_{n}\right)=0, \quad x_{n}>0, \\
\left.\mathrm{~B}_{w}\left(x^{\prime}, 0, \xi^{\prime}, D_{n}\right) u\left(x_{n}\right)\right|_{x_{n}=0}=d,
\end{array}\right.
$$

where $d \in \mathbb{C}^{v}, D_{n}=\partial^{\mathbf{1}_{n}} / i$ and $i$ is the imaginary unit. Hence A and B are some linear operators, and after fixing some $x^{\prime}$ and $\xi^{\prime}$ we may interpret them as

$$
\begin{aligned}
& \mathrm{A}_{w}: C^{\infty}\left(\mathbb{R} \times \mathbb{C}^{m}\right) \longrightarrow C^{\infty}\left(\mathbb{R} \times \mathbb{C}^{m}\right) ; \\
& \mathrm{B}_{w}: C^{\infty}\left(\mathbb{R} \times \mathbb{C}^{m}\right) \longrightarrow \mathbb{C}^{v} .
\end{aligned}
$$

Lemma 4.1 (see [3]). Suppose that A is DN-elliptic. Then

$$
\operatorname{dim}\left(\operatorname{ker}\left(\mathrm{A}_{w}\left(\xi^{\prime}, D_{n}\right)\right) \cap \mathfrak{M}_{+}\right)=v .
$$

Definition 4.1. The boundary operator (3.2) satisfies the SL-condition if the initial value problem (4.1) has a unique solution in $\mathfrak{M}_{+}$for all $d$ and for all $\xi^{\prime} \neq 0$.

Consider a boundary problem

$$
\begin{cases}A y=f, & x \in \Omega, \\ B y=g, & x \in \Gamma .\end{cases}
$$


Definition 4.2. The square boundary problem (4.2) is DN-elliptic, if

(i) the operator $A$ is DN-elliptic;

(ii) the operator $B$ satisfies the SL-condition.

Now it turns out that these conditions guarantee that the boundary problem is well posed in the following sense. Let us consider a BV-problem $\Phi=(A, B)$ with weight vectors $s, t$ and $r$. Let us also choose some $a$ such that

$$
a \geqslant-\min t_{j}, \quad a \geqslant \max s_{i}, \quad a>\max r_{i}+1 / 2 .
$$

Then one can show that $\Phi$ is a bounded operator if we choose the following Sobolev spaces [4]:

$$
\Phi=(A, B): H_{a+t}\left(\varepsilon_{0}\right) \longrightarrow H_{a-s}\left(\varepsilon_{1}\right) \times H_{a-r-1 / 2}\left(g_{1}\right) .
$$

Moreover, one has the following result.

Theorem 4.1 (see [4]). Let conditions (4.3) be satisfied. The following statements are equivalent.

(i) The boundary problem (4.2) is DN-elliptic.

(ii) The operator $\Phi$ in (4.4) is Fredholm and the following a priori estimate holds:

$$
\|y\|_{a+t} \leqslant C\left(\|f\|_{a-s}+\|g\|_{a-r-1 / 2}+\|y\|_{0}\right) .
$$

If the solution is unique, the last term on the right can be omitted.

Recall that an operator is Fredholm if it has a finite-dimensional kernel and cokernel, and its image is closed.

\subsection{Algebraic formulation of the Shapiro-Lopatinskij condition}

It is evident that in general it is very hard to check the SL-condition using the definition directly. Agmon, Douglis and Nirenberg $[2,3]$ proposed a criterion to simplify the verification of the SL-condition; see also [4] for a discussion.

Consider a DN-elliptic square operator $A$. At the fixed boundary point $\left(x^{\prime}, 0\right) \in \Gamma$ we write for simplicity $\mathrm{A}_{w}$ and $\mathrm{B}_{w}$ instead of $\mathrm{A}_{w}\left(x^{\prime}, 0, \xi^{\prime}, \zeta\right)$ and $\mathrm{B}_{w}\left(x^{\prime}, 0, \xi^{\prime}, \zeta\right)$ when the meaning is clear from the context.

Let us fix some $\xi^{\prime} \neq 0$ and let $\zeta_{1}, \ldots, \zeta_{v}$ be all the roots of the characteristic polynomial $p_{\mathrm{A}} \in \mathbb{R}[\zeta]$ lying in the upper half of the complex plane. We set

$$
p_{\mathrm{A}}^{+}=\left(\zeta-\zeta_{1}\right) \ldots\left(\zeta-\zeta_{\nu}\right)=\sum_{j=0}^{\nu} b_{j} \zeta^{j}
$$

and we introduce the polynomials $p_{l}^{+}, l=1, \ldots, v$, as in (2.2).

The traditional formulation of a criterion which implies the SL-condition is as follows.

THEOREM 4.2 (see $[3,4])$. The SL-condition is satisfied at a fixed boundary point if and only if for all $\xi^{\prime} \neq 0$ the rows of the matrix

$$
\mathrm{B}_{w} \operatorname{adj}\left(\mathrm{A}_{w}\right)
$$

are linearly independent modulo the polynomial $p_{\mathrm{A}}^{+}$. 
REMARK 4.1. This criterion was originally formulated using symbol matrices with imaginary units, but that formulation is equivalent to our formulation. To see this, let us first note that the polynomial $p_{\mathrm{A}}^{+}$does not depend on this choice. Then, dividing all elements of $\mathrm{B}_{w} \operatorname{adj}\left(\mathrm{A}_{w}\right)$ by $p_{\mathrm{A}}^{+}$, it is seen that the rank of the matrix of remainders does not depend on this choice either.

Now we would like to improve this criterion. To this end, we need to formulate some preliminary results.

LEMMA 4.2. There is an element $\left(\operatorname{adj}\left(\mathrm{A}_{w}\right)\right)_{i, j}$ of $\operatorname{adj}\left(\mathrm{A}_{w}\right)$ which is not divisible by the polynomial $p_{\mathrm{A}}^{+}$.

Proof. Suppose that $\operatorname{adj}\left(\mathrm{A}_{w}\right)=p_{\mathrm{A}}^{+} C$. On the other hand, by the property of adjoint matrix we $g e t \operatorname{det}\left(\operatorname{adj}\left(\mathrm{A}_{w}\right)\right)=p_{\mathrm{A}}^{m-1}$. Thus, $\left(p_{\mathrm{A}}^{+}\right)^{m} \operatorname{det}(C)=p_{\mathrm{A}}^{m-1}$. But this contradicts the definition of $p_{\mathrm{A}}^{+}$.

Let us denote by $v$ some column of the matrix $\operatorname{adj}\left(\mathrm{A}_{w}\right)$ which is nonzero modulo $p_{\mathrm{A}}^{+}$. We set

$$
\omega^{l}\left(x_{n}\right)=\frac{1}{2 \pi i} \oint_{\gamma_{+}} \frac{v(\zeta) p_{l}^{+}(\zeta) e^{i \zeta x_{n}}}{p_{\mathrm{A}}^{+}(\zeta)} d \zeta, \quad l=1, \ldots, v
$$

where $\gamma_{+}$is a simple closed curve oriented counterclockwise in the upper half of the complex plane surrounding all the roots of the polynomial $p_{A}^{+}$, and we let $\omega$ be a matrix with columns $\omega^{l}$.

Lemma 4.3. The columns of $\omega$ are a basis of the space $\operatorname{ker}\left(\mathrm{A}_{w}\left(D_{n}\right)\right) \cap \mathfrak{M}_{+}$.

Proof. First note that by the property of adjoint matrix, all elements of the vector $\mathrm{A}_{w} v$ are divisible by the polynomial $p_{A}^{+}$. Thus by the Cauchy integral theorem we get $\mathrm{A}_{w}\left(D_{n}\right) w^{l}\left(x_{n}\right)=0$. Since $\Re(i \zeta)<0$, we see that the columns of $w$ belong to $\operatorname{ker}\left(\mathrm{A}_{w}\left(D_{n}\right)\right) \cap \mathfrak{M}_{+}$. Lemma 2.4 implies that the vectors $\omega^{l}\left(x_{n}\right), l=1, \ldots, v$, are linearly independent. From Lemma 4.1 we find that $\operatorname{dim}\left(\operatorname{ker}\left(\mathrm{A}_{w}\left(D_{n}\right)\right) \cap \mathfrak{M}_{+}\right)=v$. As we have $v$ linearly independent vectors, they are the basis of $\operatorname{ker}\left(\mathrm{A}_{w}\left(D_{n}\right)\right) \cap \mathfrak{M}_{+}$.

Let us now consider the vector $h=\mathrm{B}_{w} v$. Dividing each element of $h$ by the polynomial $p_{\mathrm{A}}^{+}$, we get

$$
h=q p_{\mathrm{A}}^{+}+\mathrm{h} \quad \text { where } \mathrm{h}=\sum_{\tau=0}^{\nu-1} \mathrm{~h}^{\tau} \zeta^{\tau}
$$

Let us introduce the matrix $\mathscr{H}$ :

$$
\mathscr{H}=\left(\mathrm{h}^{0}, \ldots, \mathrm{h}^{\nu-1}\right)
$$

LEMma 4.4. The following equality holds:

$$
\left.\mathrm{B}_{w}\left(D_{n}\right) \omega\left(x_{n}\right)\right|_{x_{n}=0}=\mathscr{H} .
$$


Proof. Using Lemma 2.3 we have

$$
\begin{aligned}
\left.\mathrm{B}_{w}\left(D_{n}\right) \omega^{l}\left(x_{n}\right)\right|_{x_{n}=0} & =\frac{1}{2 \pi i} \oint_{\gamma_{+}} \frac{\mathrm{B}_{w}(\zeta) v(\zeta) p_{l}^{+}(\zeta)}{p_{\mathrm{A}}^{+}(\zeta)} d \zeta \\
& =\sum_{\tau=0}^{\nu-1} \mathrm{~h}^{\tau} \frac{1}{2 \pi i} \oint_{\gamma_{+}} \frac{\zeta^{\tau} p_{l}^{+}(\zeta)}{p_{\mathrm{A}}^{+}(\zeta)} d \zeta \\
& =\mathrm{h}^{l-1}, \quad l=1, \ldots, \nu .
\end{aligned}
$$

Finally, we get the following improvement of the algebraic criterion for checking the SL-condition.

THEOREM 4.3. The following statements are equivalent.

(SL) The SL-condition is satisfied at a fixed boundary point.

(SL1) For any $\xi^{\prime} \neq 0$, there is a column $v$ of $\operatorname{adj}\left(\mathrm{A}_{w}\right)$ that is nonzero modulo the polynomial $p_{\mathrm{A}}^{+}$such that the elements of the vector $h=\mathrm{B}_{w} v$ are linearly independent modulo $p_{\mathrm{A}}^{+}$.

(SL2) For any $\xi^{\prime} \neq 0$, there is a column $v$ of $\operatorname{adj}\left(\mathrm{A}_{w}\right)$ that is nonzero modulo the polynomial $p_{\mathrm{A}}^{+}$such that the following condition is fulfilled: there are no numbers $c_{i}$ with at least one of them nonzero such that

$$
c_{1} \mathrm{~h}_{1}+\ldots+c_{v} \mathrm{~h}_{v}=0,
$$

where $\mathrm{h}_{i}, i=1, \ldots, v$, are the elements of $\mathrm{h}$ defined in (4.5).

(SL3) For any $\xi^{\prime} \neq 0$, there is a column $v$ of $\operatorname{adj}\left(\mathrm{A}_{w}\right)$ that is nonzero modulo the polynomial $p_{\mathrm{A}}^{+}$such that $\operatorname{rank}(\mathscr{H})=v$ where $\mathscr{H}$ is defined by (4.6).

Proof. The conditions (SL1), (SL2) and (SL3) are easily seen to be equivalent.

$(\mathrm{SL3}) \Longrightarrow(\mathrm{SL})$ : Let us suppose that the condition (SL3) is fulfilled, and fix some $\xi^{\prime} \neq 0$. Let $v$ be a nonzero modulo $p_{\mathrm{A}}^{+}$column of $\operatorname{adj}\left(\mathrm{A}_{w}\right) \operatorname{such}$ that $\operatorname{rank}(\mathscr{H})=v$. Since we have $v$ boundary conditions, we get $\operatorname{rank}(\mathscr{H})=\operatorname{rank}(\mathscr{H}, d)=v$ for any $d$. Thus, the system $\mathscr{H} c=d$ has a solution. Using this vector $c$ we construct the following function $u\left(x_{n}\right)=\omega\left(x_{n}\right) c$. Lemma 4.3 implies that $u \in \operatorname{ker}\left(\mathrm{A}_{w}\left(D_{n}\right)\right) \cap \mathfrak{M}_{+}$. By Lemma 4.4 we get

$$
\left.\mathrm{B}_{w}\left(D_{n}\right) u\left(x_{n}\right)\right|_{x_{n}=0}=\mathscr{H} c=d .
$$

So the solution to problem (4.1) exists.

Let us now prove the uniqueness of the solution of (4.1). Suppose that there is a nonzero solution $u \in \operatorname{ker}\left(\mathrm{A}_{w}\left(D_{n}\right)\right) \cap \mathfrak{M}_{+}$which satisfies $\left.\mathrm{B}_{w}\left(D_{n}\right) u\left(x_{n}\right)\right|_{x_{n}=0}=0$. Since the columns of $\omega$ are the basis of $\operatorname{ker}\left(\mathrm{A}_{w}\left(D_{n}\right)\right) \cap \mathfrak{M}_{+}$, there is a $c \neq 0$ such that $u\left(x_{n}\right)=\omega\left(x_{n}\right) c$. Then $\left.\mathrm{B}_{w}\left(D_{n}\right) u\left(x_{n}\right)\right|_{x_{n}=0}=\mathscr{H} c=0$. But this contradicts (SL3).

$(\mathrm{SL}) \Longrightarrow(\mathrm{SL3})$ : Suppose now that the SL-condition is fulfilled. The SL-condition is equivalent to the following condition: for all $\xi^{\prime} \neq 0$,

$$
\operatorname{ker}\left(\mathrm{B}_{w}\right)=\{0\}, \quad \operatorname{im}\left(\mathrm{B}_{w}\right)=\mathbb{C}^{v},
$$

where $\mathrm{B}_{w}: \operatorname{ker}\left(\mathrm{A}_{w}\left(D_{n}\right)\right) \cap \mathfrak{M}_{+} \rightarrow \mathbb{C}^{v}$ is a linear operator. Fix some $\xi^{\prime} \neq 0$ and let $v$ be a nonzero modulo $p_{\mathrm{A}}^{+}$column of $\operatorname{adj}\left(\mathrm{A}_{w}\right)$. Then according to Lemma 4.3 the columns of $\omega\left(x_{n}\right)$ are a basis of space $\operatorname{ker}\left(\mathrm{A}_{w}\left(D_{n}\right)\right) \cap \mathfrak{M}_{+}$. Since $\mathrm{B}_{w}$ is a linear operator, $\operatorname{ker}\left(\mathrm{B}_{w}\right)=\{0\}$ and $\operatorname{dim}\left(\operatorname{im}\left(\mathrm{B}_{w}\right)\right)=v$, we find that the columns of $\left.\mathrm{B}_{w}\left(D_{n}\right) \omega\left(x_{n}\right)\right|_{x_{n}=0}=\mathscr{H}$ are a basis of the $\operatorname{space} \operatorname{im}\left(B_{w}\right)$. Hence $\operatorname{rank}(\mathscr{H})=v$. 
REMARK 4.2. Note that if there is one column $v$ of $\operatorname{adj}\left(\mathrm{A}_{w}\right)$ that is nonzero modulo the polynomial $p_{\mathrm{A}}^{+} \operatorname{such}$ that $\operatorname{rank}(\mathscr{H})=v$, then this property is true for any column of $\operatorname{adj}\left(\mathrm{A}_{w}\right)$ that is nonzero modulo $p_{\mathrm{A}}^{+}$.

REMARK 4.3. The difference between our formulation and the result of Agmon, Douglis and Nirenberg [3] is that they considered the whole adjoint in the criterion, while it is only necessary to take one column which is nonzero modulo $p_{\mathrm{A}}^{+}$.

\subsection{Computational test for checking the SL-condition in the two-variable case}

The idea of the formulations of the criterion for the SL-condition is to consider $\xi^{\prime}$ as a 'parameter' (to fix it), and not as a 'variable'. Hence in principle we should use the test for each $\xi^{\prime}$ separately. However, we would like to have a test which is valid for all $\xi^{\prime}$. Unfortunately, we have found such a test only in the case of two independent variables. Note that this case is already very important in PDE theory.

The main difficulty is to compute $p_{A}^{+}$. In the two-variable case we can use the fact that $p_{\mathrm{A}}$ is homogeneous to factor it, but this idea does not work in the case of more variables. For example if $p_{\mathrm{A}}=\xi_{1}^{2}+\zeta^{2}$, then we can easily factor this using the factors of $\zeta^{2}+1$, so the problem reduces to the factorisation of the polynomial of one variable. However, if $p_{\mathrm{A}}=\xi_{1}^{2}+\xi_{2}^{2}+\zeta^{2}$, then we cannot proceed in the same way, even though formally we can factor this as

$$
p_{\mathrm{A}}=\left(\zeta-i \sqrt{\xi_{1}^{2}+\xi_{2}^{2}}\right)\left(\zeta+i \sqrt{\xi_{1}^{2}+\xi_{2}^{2}}\right) .
$$

Consider the system (2.11) in two variables. Then the characteristic polynomial $p_{\mathrm{A}}$ is a homogeneous polynomial in two variables $\xi=\left(\xi_{1}, \zeta\right)$ of degree $2 v$. Dehomogenising $p_{\mathrm{A}}$ (that is, setting $\xi_{1}=1$ ), we get a polynomial $\hat{p}_{\mathrm{A}}$. In the same way we denote the dehomogenised symbol matrices of $\mathrm{A}_{w}$ and $\mathrm{B}_{w}$ by $\hat{\mathrm{A}}_{w}$ and $\hat{\mathrm{B}}_{w}$. It is easy to see that $\operatorname{det}\left(\hat{\mathrm{A}}_{w}\right)=\hat{p}_{\mathrm{A}}$.

Let $\mathbb{K}(a)$ be a splitting field for $\hat{p}_{\mathrm{A}}$ with minimal polynomial $p_{\min }$ of degree $\ell$. Hence $\mathbb{K}(a)$ is a $\mathbb{K}$-vector space of dimension $\ell$ with basis $\left\{1, a, a^{2}, \ldots, a^{\ell-1}\right\}$. Let $\hat{a} \in \mathbb{C}$ be a root of the polynomial $p_{\min }$. It is easily seen that the map

$$
\iota: \mathbb{K}(a) \longrightarrow \mathbb{C}, \quad \iota\left(\sum_{i=0}^{\ell-1} c_{i} a^{i}\right)=\sum_{i=0}^{\ell-1} c_{i} \hat{a}^{i},
$$

is an injective homomorphism and it induces the ring homomorphism

$$
\tilde{\iota}: \mathbb{K}(a)[\zeta] \longrightarrow \mathbb{C}[\zeta], \quad \tilde{\iota}\left(\sum_{i=0}^{\tau-1} b_{i} \zeta^{i}\right)=\sum_{i=0}^{\tau-1} \iota\left(b_{i}\right) \zeta^{i}
$$

Also, $\tilde{\iota}$ is injective and it preserves the degree of a polynomial. The notation $\iota(C)$ where $C$ is a matrix means that we apply the map $\iota$ to each element of $C$. Likewise, $\tilde{\imath}(C)$ where $C$ is a matrix means that we apply the map $\tilde{\iota}$ to each element of $C$.

Let $\rho_{1}, \ldots, \rho_{v} \in \mathbb{K}(a)$ be the roots of $\hat{p}_{\mathrm{A}}$ such that $\iota\left(\rho_{1}\right), \ldots, \iota\left(\rho_{v}\right)$ are in the upper half of the complex plane, and let

$$
\hat{p}_{\mathrm{A}}^{+}=\left(\zeta-\rho_{1}\right) \ldots\left(\zeta-\rho_{\nu}\right) \in \mathbb{K}(a)[\zeta] .
$$

Further, let

$$
\tilde{p}_{\mathrm{A}}^{+}=\left(\zeta-\iota\left(\rho_{1}\right) \xi_{1}\right) \ldots\left(\zeta-\iota\left(\rho_{\nu}\right) \xi_{1}\right), \quad \tilde{p}_{\mathrm{A}}^{-}=\left(\zeta-\overline{\imath\left(\rho_{1}\right)} \xi_{1}\right) \ldots\left(\zeta-\overline{\iota\left(\rho_{\nu}\right)} \xi_{1}\right)
$$


Then evidently $p_{\mathrm{A}}=\tilde{p}_{\mathrm{A}}^{+} \tilde{p}_{\mathrm{A}}^{-}$and for a fixed $\xi^{\prime} \neq 0$,

$$
p_{\mathrm{A}}^{+}= \begin{cases}\tilde{p}_{\mathrm{A}}^{+}, & \text {if } \xi_{1}>0 \\ \tilde{p}_{\mathrm{A}}^{-}, & \text {if } \xi_{1}<0\end{cases}
$$

We will show that it is in fact sufficient to work with $\hat{p}_{\mathrm{A}}^{+}$. To this end we will need the following fact. For divisions in $\mathbb{C}\left[\xi_{1}, \zeta\right]$ we use lexicographic ordering with $\zeta>\xi_{1}$.

LEMMA 4.5. There is an element $\hat{d}$ of $\operatorname{adj}\left(\hat{\mathrm{A}}_{w}\right)$ such that

(i) $\hat{d}$ is not divisible by $\hat{p}_{\mathrm{A}}^{+}$;

(ii) $\hat{d}$ is not divisible by $\tilde{\imath}\left(\hat{p}_{\mathrm{A}}^{+}\right)$;

(iii) the corresponding element $d$ of $\operatorname{adj}\left(\mathrm{A}_{w}\right)$ is not divisible by $\tilde{p}_{\mathrm{A}}^{+}$;

(iv) for any fixed $\xi_{1} \neq 0$ the corresponding element $d$ of $\operatorname{adj}\left(\mathrm{A}_{w}\right)$ is not divisible by $p_{\mathrm{A}}^{+}$.

Proof. Lemma 4.2 immediately implies the first statement. Hence there are polynomials $q, r \in \mathbb{K}(a)[\zeta]$ such that $\hat{d}=q \hat{p}_{\mathrm{A}}^{+}+r, r \neq 0$ and $\operatorname{deg} r<v$, and consequently $\hat{d}=\tilde{\iota}(\hat{d})=$ $\tilde{l}(q) \tilde{l}\left(\hat{p}_{\mathrm{A}}^{+}\right)+\tilde{l}(r)$. Since $\tilde{\iota}$ is injective and it preserves the degree of a polynomial, $\tilde{l}(r)$ is a nonzero remainder, which proves the second statement.

Then let us divide $d$ by $\tilde{p}_{\mathrm{A}}^{+}$, which yields $d=q \tilde{p}_{\mathrm{A}}^{+}+r$. But this is simply a homogenised version of $\hat{d}=\tilde{\imath}(q) \tilde{\imath}\left(\hat{p}_{\mathrm{A}}^{+}\right)+\tilde{\imath}(r)$. Hence $r$ cannot be zero, which proves the third statement.

Since $d$ is not divisible by $\tilde{p}_{\mathrm{A}}^{+}$, there are polynomials $q, r \in \mathbb{C}\left[\xi_{1}, \zeta\right]$ such that $d=$ $q \tilde{p}_{\mathrm{A}}^{+}+r$ where $r \neq 0$ and none of the monomials of $r$ is divisible by $\zeta^{v}$. Since $r$ is homogeneous, it will remain nonzero if we substitute some nonzero real number for $\xi_{1}$. Hence the fourth statement is valid for $\xi_{1}>0$ because in that case $p_{A}^{+}=\tilde{p}_{A}^{+}$. Then if $\xi_{1}<0$, it is easy to see that $d=\bar{q} p_{\mathrm{A}}^{+}+\bar{r}$. Hence in this case also, the remainder $\bar{r}$ is nonzero.

Suppose that $\hat{d}$ is in the $j$ th column of $\operatorname{adj}\left(\mathrm{A}_{w}\right)$. We denote by $\hat{v}$ the $j$ th column of $\operatorname{adj}\left(\hat{\mathrm{A}}_{w}\right)$, and by $v$ the $j$ th column of $\operatorname{adj}\left(\mathrm{A}_{w}\right)$.

Then dividing all elements of $\hat{h}=\hat{\mathrm{B}}_{w} \hat{v}$ by $\hat{p}_{\mathrm{A}}^{+}$gives $\hat{h}=\hat{q} \hat{p}_{\mathrm{A}}^{+}+\hat{\mathrm{h}}$, where $\operatorname{deg} \hat{\mathrm{h}}_{i}<v$. Setting

$$
\hat{\mathrm{h}}=\sum_{\tau=0}^{\nu-1} \hat{\mathrm{h}}^{\tau} \zeta^{\tau}
$$

we introduce the matrix

$$
\hat{\mathscr{H}}=\left(\hat{\mathrm{h}}^{0}, \ldots, \hat{\mathrm{h}}^{\nu-1}\right)
$$

whose elements are in $\mathbb{K}(a)$. In the same way we can divide $\hat{h}$ by $\tilde{\imath}\left(\hat{p}_{\mathrm{A}}^{+}\right)$and construct the analog of the matrix $\hat{\mathscr{H}}$. It is clear that the result will be $\iota(\hat{\mathscr{H}})$.

Set $\tilde{h}=\mathrm{B}_{w} v$. The $i$ th component of $\tilde{h}$ is a homogeneous polynomial in $\xi=\left(\xi^{\prime}, \zeta\right)$ of degree $r_{i}+2 v-s_{j}\left(v\right.$ is the $j$ th column of $\left.\operatorname{adj}\left(\mathrm{A}_{w}\right)\right)$. Using the division algorithm, we get $\tilde{h}=\tilde{q} \tilde{p}_{\mathrm{A}}^{+}+\tilde{\mathrm{h}}$. Since the components of $\tilde{q}$ and $\tilde{h}$ are homogeneous polynomials, the $i$ th component of $\tilde{h}$ is also a homogeneous polynomial of the degree $r_{i}+2 v-s_{j}$. Setting now

$$
\tilde{\mathrm{h}}=\sum_{\tau=0}^{\nu-1} \tilde{\mathrm{h}}^{\tau} \zeta^{\tau} \quad \text { and } \quad \tilde{\mathscr{H}}=\left(\tilde{\mathrm{h}}^{0}, \ldots, \tilde{\mathrm{h}}^{\nu-1}\right) \text {, }
$$


we readily see that $\tilde{\mathrm{h}}_{i}^{\tau}=\iota\left(\hat{\mathrm{h}}_{i}^{\tau}\right) \xi_{1}^{r_{i}+2 v-s_{j}-\tau}$, and that

$$
\operatorname{det}(\tilde{\mathscr{H}})=\xi_{1}^{\nu\left(2 v-s_{j}-(v-1) / 2\right)+r_{1}+\ldots+r_{v}} \operatorname{det}(\iota(\hat{\mathscr{H}})) .
$$

Finally, we compute $h=\mathrm{B}_{w} v$ with some fixed $\xi_{1} \neq 0$. Dividing $h$ by $p_{\mathrm{A}}^{+}$and constructing the corresponding matrix $\mathscr{H}$ as above, we obtain for any fixed $\xi_{1} \neq 0$,

$$
\mathscr{H}= \begin{cases}\tilde{\mathscr{H}}, & \text { if } \xi_{1}>0, \\ \tilde{\mathscr{H}}, & \text { if } \xi_{1}<0 .\end{cases}
$$

Now we can formulate a computational test for checking the SL-condition.

THEOREM 4.4. An operator $(A, B)$ satisfies the SL-condition if and only if $\operatorname{det}(\hat{\mathscr{H}}) \neq 0$ where $\hat{\mathscr{H}}$ is a matrix as defined in (4.12).

Proof. The condition (SL3) of Theorem 4.3 is equivalent to $\operatorname{det}(\mathscr{H}) \neq 0$ for all $\xi_{1} \neq 0$. By (4.14) this is equivalent to $\operatorname{det}(\tilde{\mathscr{H}}) \neq 0$ for all $\xi_{1} \neq 0$. Using (4.13) we obtain the condition $\operatorname{det}(\iota(\hat{\mathscr{H}})) \neq 0$. But since $\iota$ is an injective homomorphism, this is equivalent to $\operatorname{det}(\hat{\mathscr{H}}) \neq 0$.

Note that this formulation of the SL-condition can effectively be tested using Gröbner basis techniques, for example with the program Singular [13]. (See Appendix A.1, Appendix A.2 and Appendix A.3 for the appropriate commands in Singular).

EXAMPLE 4.1. Let us consider the following system:

$$
A y=\left\{\begin{array}{l}
y_{20}^{1}+y_{02}^{1}+y_{20}^{3}=0, \\
2 y_{10}^{1}+y_{01}^{2}=0, \\
y_{10}^{2}+y_{01}^{3}=0,
\end{array} \quad \text { in } \mathbb{R}_{+}^{2}=\left\{x \in \mathbb{R}^{2}: x_{2}>0\right\}\right.
$$

Taking weights $t_{1}=t_{2}=t_{3}=2$ and $s_{1}=0, s_{2}=s_{3}=-1$, we get the weighted principal symbol of (4.15):

$$
A_{w}=\left(\begin{array}{ccc}
\zeta^{2}+\xi_{1}^{2} & 0 & \xi_{1}^{2} \\
2 \xi_{1} & \zeta & 0 \\
0 & \xi_{1} & \zeta
\end{array}\right)
$$

Since $p_{\mathrm{A}}=\operatorname{det}\left(\mathrm{A}_{w}\right)=\zeta^{4}+\zeta^{2} \xi_{1}^{2}+2 \xi_{1}^{4}$, the system (4.15) is $D N$-elliptic. We set $\hat{p}_{\mathrm{A}}=$ $\zeta^{4}+\zeta^{2}+2$

Computing with Singular as discussed above, we get the following statements:

- the splitting field of $\hat{p}_{\mathrm{A}}$ is $\mathbb{Q}(\alpha)$ with a minimal polynomial

$$
p_{\min }=\alpha^{8}+10 \alpha^{6}+5 \alpha^{4}-100 \alpha^{2}+2116
$$

- the roots of the polynomial $\hat{p}_{\mathrm{A}}$ are

$$
\begin{aligned}
& \rho_{1}=-\rho_{2}=-\frac{7}{105432} \alpha^{7}-\frac{265}{52716} \alpha^{5}-\frac{3485}{105432} \alpha^{3}-\frac{6883}{17572} \alpha ; \\
& \rho_{3}=-\rho_{4}=\frac{7}{52716} \alpha^{7}+\frac{265}{26358} \alpha^{5}+\frac{3485}{52716} \alpha^{3}-\frac{1903}{8786} \alpha .
\end{aligned}
$$

Now we find numerically, using Singular, the roots of the polynomial $p_{\min }$ in $\mathbb{C}$ :

$$
\begin{aligned}
& \alpha_{1}=-\overline{\alpha_{2}}=-\alpha_{3}=\overline{\alpha_{4}} \approx-0.676+i 2.935 ; \\
& \alpha_{5}=-\alpha_{6}=\overline{\alpha_{7}}=-\overline{\alpha_{8}} \approx-2.028+i 0.978
\end{aligned}
$$


Substituting $\alpha_{1}$ in the roots of polynomial $\hat{p}_{\mathrm{A}}$ we obtain

$$
\rho_{1}\left(\alpha_{1}\right)=-\rho_{2}\left(\alpha_{1}\right)=-\overline{\rho_{3}\left(\alpha_{1}\right)}=\overline{\rho_{4}\left(\alpha_{1}\right)} \approx 0.676-i 0.978 .
$$

So for the primitive element $\alpha_{1}$, the roots $\rho_{2}$ and $\rho_{4}$ are in the upper half of the complex plane. Hence we get

$$
\hat{p}_{\mathrm{A}}^{+}=\left(\zeta-\rho_{2}\right)\left(\zeta-\rho_{4}\right) \in \mathbb{Q}(\alpha)[\zeta]
$$

Let us compute the adjoint matrix of $\hat{\mathrm{A}}_{w}$. We get

$$
\operatorname{adj}\left(\hat{\mathrm{A}}_{w}\right)=\left(\begin{array}{ccc}
\zeta^{2} & 1 & -\zeta \\
-2 \zeta & \zeta^{3}+\zeta & 2 \\
2 & -\zeta^{2}-1 & \zeta^{3}+\zeta
\end{array}\right)
$$

Let $\hat{v}$ be the first column of $\operatorname{adj}\left(\hat{\mathrm{A}}_{w}\right)$; it is nonzero modulo $\hat{p}_{\mathrm{A}}^{+}$. We will carry out the SL-test symbolically in the polynomial ring $\mathbb{Q}(\alpha)[\zeta]$. Since $\operatorname{deg} \hat{p}_{\mathrm{A}}=4$, we need to impose two boundary conditions on the system (4.15). First consider the following boundary conditions:

$$
B_{1} y=\left\{\begin{array}{l}
y^{1}+y^{3}=0, \\
y^{2}=0,
\end{array} \quad \text { on } \partial \mathbb{R}_{+}^{2} .\right.
$$

So the principal symbol of $B_{1}$ is

$$
\mathrm{B}_{1}=\left(\begin{array}{lll}
1 & 0 & 1 \\
0 & 1 & 0
\end{array}\right) \quad \text { and hence } \quad \hat{\mathrm{B}}_{1} \hat{v}=\left(\begin{array}{c}
\zeta^{2}+2 \\
-2 \zeta
\end{array}\right)
$$

Reducing this vector with respect to $\hat{p}_{\mathrm{A}}^{+}$, we get

$$
\hat{\mathrm{h}}=\left(\begin{array}{c}
\beta_{1} \zeta+\beta_{0} \\
-2 \zeta
\end{array}\right), \quad \hat{\mathscr{H}}=\left(\begin{array}{cc}
\beta_{0} & \beta_{1} \\
0 & -2
\end{array}\right)
$$

where

$$
\begin{aligned}
& \beta_{1}=-\frac{7}{105432} \alpha^{7}-\frac{265}{52716} \alpha^{5}-\frac{3485}{105432} \alpha^{3}+\frac{10689}{17572} \alpha \\
& \beta_{0}=\frac{1}{382} \alpha^{6}+\frac{15}{764} \alpha^{4}-\frac{123}{764} \alpha^{2}+\frac{579}{382} .
\end{aligned}
$$

Since $\operatorname{det}(\hat{\mathscr{H}})=-2 \beta_{0} \neq 0$, we see that $B_{1}$ satisfies the SL-condition.

Let us now impose the following boundary conditions on the system (4.15):

$$
B_{2} y=\left\{\begin{array}{l}
y^{1}=0 \\
y_{01}^{2}=0
\end{array}\right.
$$

Computing $\left(\hat{\mathrm{B}}_{2}\right)_{w} \hat{v}$ and reducing with respect to $\hat{p}_{\mathrm{A}}^{+}$, we have

$$
\hat{\mathrm{h}}=\left(\begin{array}{c}
\beta_{1} \zeta+b_{0} \\
-2 \beta_{1} \zeta-2 b_{0}
\end{array}\right), \quad \hat{\mathscr{H}}=\left(\begin{array}{cc}
b_{0} & \beta_{1} \\
-2 b_{0} & -2 \beta_{1}
\end{array}\right),
$$

where

$$
b_{0}=\frac{1}{382} \alpha^{6}+\frac{15}{764} \alpha^{4}-\frac{123}{764} \alpha^{2}-\frac{185}{382} .
$$

Now $\operatorname{det}(\hat{\mathscr{H}})=0$, and hence $B_{2}$ does not satisfy the SL-condition. 


\section{Overdetermined elliptic boundary problems (general theory)}

\subsection{Differential boundary operators}

Suppose that the system $(2.11)$ is overdetermined $(k \geqslant m)$. Since any linear overdetermined DN-elliptic system becomes elliptic during its completion to involutive form [15], we can assume without loss of generality that the system (2.11) is elliptic with the principal symbol A. Hence we consider now a boundary problem

$$
\begin{cases}A y=f, & x \in \Omega, \\ B y=g, & x \in \Gamma,\end{cases}
$$

where $A$ is a $q$ th-order elliptic differential operator and $B$ is of size $\tilde{v} \times m$; we write this as $B=\left(B_{1}, \ldots, B_{\tilde{v}}\right)$, and we denote the order of the operator $B_{i}$ by $r_{i}$. We will always suppose that $\tilde{v} \geqslant v$ because otherwise the problem would not be Fredholm: the kernel of the BV-operator $\Phi_{0}=(A, B)$ would be infinite-dimensional.

Note that now we still need a nontrivial weight vector for $B$, but for $s$ and $t$ we can take $t_{j}=0$ and $s_{i}=q$. Consequently, it is natural to consider $\Phi_{0}$ as operating in the following Sobolev spaces:

$$
\Phi_{0}=(A, B): H_{a}\left(\varepsilon_{0}\right) \longrightarrow H_{a-q}\left(\varepsilon_{1}\right) \times H_{a-r-1 / 2}\left(\mathscr{g}_{1}\right),
$$

where $a$ is some appropriately chosen number and $r=\left(r_{1}, \ldots, r_{\tilde{v}}\right)$. To study the wellposedness of this boundary problem we need to construct a compatibility operator for $\Phi_{0}$. It turns out that this can be done by using a certain equivalent first-order system.

DEFINITION 5.1. A differential operator $A: \mathrm{S}\left(\varepsilon_{0}\right) \rightarrow \mathrm{S}\left(\varepsilon_{1}\right)$ is called normalised if

(i) $A$ is a first-order operator;

(ii) $A$ is involutive;

(iii) the principal symbol $A: T^{*} \Omega \otimes \varepsilon_{0} \rightarrow \varepsilon_{1}$ is surjective.

Condition (iii) means that there are no (explicit or implicit) algebraic (that is, nondifferential) relations between dependent variables in the system. If such relations exist, then we may use them to reduce the number of dependent variables. Note also that condition (iii) is equivalent to the surjectivity of the symbol matrix $M_{1}$. This follows from formula (2.12) and diagram (2.10).

DEFINITION 5.2. A DB-operator $\Phi$ is normalised if $\Phi^{11}$ is normalised and $\gamma \Phi^{21}$ contains only differentiation in directions tangent to the boundary. Likewise, a $\mathrm{BV}$-operator $(A, B)$ ) is normalised if $A$ is normalised and $B$ contains only differentiation in directions tangent to the boundary.

So the idea is to replace the original BV-operator by an equivalent normalised operator, compute its compatibility operator, and then use this to construct the compatibility operator for the original operator. To this end, we must recall some facts about compatibility operators and make more precise what is meant by 'equivalence'. Now, intuitively one thinks that equivalent systems should have the same solution spaces. However, this would be rather difficult to define precisely in a useful way. Instead, the concept of equivalence is defined with help of certain maps between bundles. We will next describe how this is done. 


\subsection{Compatibility}

Of course, we are mostly interested in differential operators, but the notion of a compatibility operator can be defined for any bundle map.

Definition 5.3. Let $\Phi_{0}: \mathcal{F}_{0} \rightarrow \mathcal{F}_{1}$ be a bundle map. The bundle map $\Phi_{1}: \mathcal{F}_{1} \rightarrow \mathcal{F}_{2}$ is a compatibility map for $\Phi_{0}$ if:

(i) $\Phi_{1} \Phi_{0}=0$;

(ii) for any bundle map $\tilde{\Phi}_{1}: \widetilde{F}_{1} \rightarrow \tilde{\mathcal{F}}_{2}$ such that $\tilde{\Phi}_{1} \Phi_{0}=0$, there is a bundle map $T: \tilde{F}_{2} \rightarrow \tilde{\mathcal{F}}_{2}$ such that $\tilde{\Phi}_{1}=T \Phi_{1}$.

This idea leads naturally to the next definition.

Definition 5.4. A complex

$$
\mathcal{C}: 0 \longrightarrow \mathcal{F}_{0} \stackrel{\Phi_{0}}{\longrightarrow} \mathcal{F}_{1} \stackrel{\Phi_{1}}{\longrightarrow} \mathcal{F}_{2} \stackrel{\Phi_{2}}{\longrightarrow} \cdots
$$

is called a compatibility complex for $\Phi_{0}$ if every map $\Phi_{i}$ for $i \geqslant 1$ is a compatibility map for $\Phi_{i-1}$.

It is rather straightforward to show that a compatibility operator exists for operators with constant coefficients. Let $\Omega \subset \mathbb{R}^{n}$ be open, and let $A_{0}: \mathrm{S}\left(\mathcal{E}_{0}\right) \rightarrow \mathrm{S}\left(\mathcal{E}_{1}\right)$ be a differential operator with constant coefficients where $\varepsilon_{0}=\Omega \times \mathbb{R}^{k_{0}}$ and $\varepsilon_{1}=\Omega \times \mathbb{R}^{k_{1}}$.

Let us consider the matrix $\tilde{\mathrm{A}}_{0}$, the full symbol of $A_{0}$ :

$$
\tilde{\mathrm{A}}_{0}=\sum_{|\mu| \leqslant q} a_{\mu} \xi^{\mu} .
$$

Note that for our purposes we do not need to add the factor $i^{|\mu|}$. Denoting by $a^{1}, \ldots, a^{k_{1}}$ the rows of $\tilde{\mathrm{A}}_{0}$, we may construct a free resolution of the module $M_{0}=\left\langle a^{1}, \ldots, a^{k_{1}}\right\rangle$ as in (2.1) as follows:

$$
0 \longrightarrow \mathbb{A}^{k_{r}} \stackrel{\tilde{\mathrm{A}}_{r}^{T}}{\longrightarrow} \mathbb{A}^{k_{r-1}} \longrightarrow \cdots
$$

$$
\cdots \stackrel{\tilde{\mathrm{A}}_{2}^{T}}{\longrightarrow} \mathbb{A}^{k_{2}} \stackrel{\tilde{\mathrm{A}}_{1}^{T}}{\longrightarrow} \mathbb{A}^{k_{1}} \stackrel{\tilde{\mathrm{A}}_{0}^{T}}{\longrightarrow} \mathbb{A}^{k_{0}} \longrightarrow \mathbb{A}^{k_{0}} / M_{0} \longrightarrow 0 .
$$

Let us denote by $A_{i}$ the differential operator corresponding to the syzygy matrix $\tilde{\mathrm{A}}_{i}$. Now we say that a complex $\mathcal{C}$ consisting of trivial bundles $\varepsilon_{i}=\Omega \times \mathbb{R}^{k_{i}}$ and operators $A_{i}$ is a Hilbert complex, if the operators $A_{i}$ are associated to the syzygy matrices of the free resolution of $\mathbb{A}$-module $M_{0}$.

THEOREM 5.1 (see [25, p. 31]). Let $\mathcal{C}$ be a complex of differential operators with constant coefficients. Then $\mathcal{C}$ is a compatibility complex for $A_{0}$ if and only if $\mathcal{C}$ is a Hilbert complex associated with the $\mathbb{A}$-module $M_{0}$.

Note that the compatibility complex which exists by the above theorem can be constructively computed using Gröbner basis techniques; see Appendix A.4. In the general case, we have the following statement.

THEOREM 5.2. Every sufficiently regular differential operator has a compatibility operator. 
See [8], [19] and [25] for more details. Note that in the above theorems there is no need to pass to the normalised operator. However, in case of $\mathrm{BV}$-operators the construction of the compatibility operator is more involved. Here we suppose at the outset that the operator is normalised, and then we discuss later how this leads to the compatibility operator in the general case.

So let us consider a normalised BV-operator $\Phi=(A, B): \mathrm{S}\left(\varepsilon_{0}\right) \rightarrow \mathrm{S}\left(\varepsilon_{1}\right) \times \mathrm{S}\left(q_{1}\right)$. We will suppose that $A$ is sufficiently regular, and will denote the compatibility operator of $A$ by $A_{1}$. Then we will need to define the tangent part of $A$, denoted by $A^{\tau}$; see [8]. The embedding of the boundary $\Gamma$ in $\Omega$ induces in the sections of the jet bundles a map $\mathrm{e}_{0}:\left.J^{1}\left(\varepsilon_{0}\right)\right|_{\Gamma} \rightarrow J^{1}\left(\left.\mathcal{E}_{0}\right|_{\Gamma}\right)$ such that

$$
\mathrm{e}_{0}\left(\left.\left(j^{1} y\right)\right|_{x}\right)=j^{1}(\gamma y)(x), \quad x \in \Gamma, y \in \mathrm{S}\left(\varepsilon_{0}\right) .
$$

We define the bundle $\varepsilon_{1}^{\tau} \rightarrow \Gamma$ by

$$
\varepsilon_{1}^{\tau}=\left.\varepsilon_{1}\right|_{\Gamma} /\left(\mathcal{A} \operatorname{ker}\left(\mathrm{e}_{0}\right)\right) .
$$

Let $\operatorname{pr}^{\tau}: \mathcal{E}_{1} \rightarrow \mathcal{E}_{1}^{\tau}$ be the projection. So we may uniquely define a map $\mathcal{A}^{\tau}$ by requiring that the following diagram commutes.

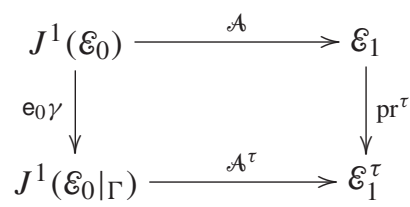

DeFinition 5.5. The differential operator $A^{\tau}: \mathrm{S}\left(\left.\varepsilon_{0}\right|_{\Gamma}\right) \rightarrow \mathrm{S}\left(\varepsilon_{1}^{\tau}\right)$ defined by $A^{\tau}=$ $\left.\mathcal{A}^{\tau} j^{1}\right|_{\Gamma}$ is called the tangent part of $A$.

It can be shown that if $A$ is normalised, then so is $A^{\tau}$; see [8]. Let us denote the compatibility operator of $A^{\tau}$ by $A_{1}^{\tau}$. Then we define a differential operator

$$
\Phi^{\tau}: \mathrm{S}^{\tau}\left(\left.\mathcal{E}_{0}\right|_{\Gamma}\right) \longrightarrow \mathrm{S}^{\tau}\left(\mathcal{E}_{1}^{\tau}\right) \times \mathrm{S}\left(\mathscr{g}_{1}\right), \quad \Phi^{\tau}(y)=\left(A^{\tau} y, B y\right) .
$$

Let $\Phi_{1}^{\tau}$ be a compatibility operator for $\Phi^{\tau}$. Note that $\Phi_{1}^{\tau}$ exists, by Theorem 5.2 , if $\Phi^{\tau}$ is sufficiently regular. If this is the case, we say that the BV-operator $\Phi$ is regular.

Note that in general $\Phi_{1}^{\tau}$ may always be written in the form $\Phi_{1}^{\tau}\left(f^{\prime}, g\right)=\left(A_{1}^{\tau} f^{\prime}, \Upsilon^{\tau}\left(f^{\prime}, g\right)\right)$, where $\Upsilon^{\tau}$ does not contain relations only between the components of $f^{\prime}$. Let us then finally define

$$
\Phi_{1}: \mathrm{S}\left(\mathscr{E}_{1}\right) \times \mathrm{S}\left(g_{1}\right) \longrightarrow \mathrm{S}\left(\mathscr{E}_{2}\right) \times \mathrm{S}\left(g_{2}\right), \quad \Phi_{1}(f, g)=\left(A_{1} f, \Upsilon^{\tau}\left(\mathrm{pr}^{\tau} f, g\right)\right)
$$

We will need the following important result [8, p. 40].

THEOREM 5.3. If $\Phi=(A, B)$ is a regular elliptic normalised BV-operator, then the DBoperator $\Phi_{1}$ defined by (5.4) is a compatibility operator for $\Phi$.

This gives a construction of the compatibility operator for the normalised BV-operator. Next we will indicate how this can be used to construct the compatibility operator in the general case.

\subsection{Equivalence of operators}

We start by giving rigorous definitions of what is meant by the 'equivalence' of operators and complexes [25]. 
Definition 5.6. Two complexes $\mathcal{C}$ and $\mathcal{C}^{\prime}$ are equivalent if the following conditions are satisfied.

(i) There are maps $M_{i}$ and $N_{i}$ such that the following diagram commutes for all $i$.

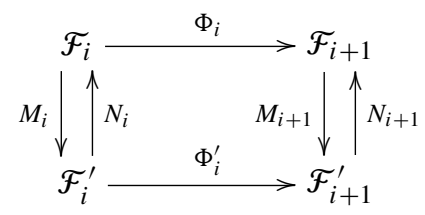

(ii) There are maps $\Psi_{i}$ and $\Psi_{i}^{\prime}$ such that for all $i$,

$$
\begin{aligned}
& \Psi_{i} \Phi_{i}+\Phi_{i-1} \Psi_{i-1}=\mathrm{id}-N_{i} M_{i} \\
& \Psi_{i}^{\prime} \Phi_{i}^{\prime}+\Phi_{i-1}^{\prime} \Psi_{i-1}^{\prime}=\mathrm{id}-M_{i} N_{i}
\end{aligned}
$$

The equivalence defined above is sometimes called homotopical or cochain equivalence.

DeFinition 5.7. Bundle maps $\Phi: \mathcal{F}_{0} \rightarrow \mathcal{F}_{1}$ and $\Phi^{\prime}: \mathcal{F}_{0}^{\prime} \rightarrow \mathcal{F}_{1}^{\prime}$ are equivalent if the complexes

$$
0 \longrightarrow \mathcal{F}_{0} \stackrel{\Phi}{\longrightarrow} \mathscr{F}_{1}
$$

and

$$
0 \longrightarrow \mathcal{F}_{0}^{\prime} \stackrel{\Phi^{\prime}}{\longrightarrow} \mathcal{F}_{1}^{\prime}
$$

are equivalent.

The concept of equivalence is important for our purposes because of the following theorems.

THEOREM 5.4. Every sufficiently regular operator A may be transformed in a finite number of steps into an equivalent normalised operator. Every DB-operator $\Phi$ whose component $\Phi^{11}$ is sufficiently regular is equivalent to a normalised $D B$-operator, and every $B V$-operator $(A, B)$ whose component $A$ is sufficiently regular is equivalent to a normalised $B V$-operator.

Then if we know a compatibility operator for some operator, we can construct a compatibility operator for an equivalent operator as follows.

THEOREM 5.5. Let $\Phi_{0}$ and $\Phi_{0}^{\prime}$ be equivalent bundle maps. If there is a compatibility complex for $\Phi_{0}^{\prime}$, then there is also a compatibility complex for $\Phi_{0}$. Moreover, their compatibility complexes are equivalent.

Proof. Here we just give the formula for constructing the first compatibility map, since we will need it below. For the details of the proof we refer to [25]. We fix some maps $M_{i}, N_{i}$ $(i=0,1), \Psi_{0}$ and $\Psi_{0}^{\prime}$ as in Definition 5.6. We set $\mathcal{F}_{2}=\mathcal{F}_{2}^{\prime} \oplus \mathcal{F}_{1}$. Then if we know the compatibility map $\Phi_{1}^{\prime}$, the compatibility map $\Phi_{1}$ is given by the formula

$$
\Phi_{1}=\left(\Phi_{1}^{\prime} M_{1}\right) \oplus\left(\mathrm{id}-N_{1} M_{1}-\Phi_{0} \Psi_{0}\right)
$$

Hence to construct a compatibility operator for the BV-operator $\Phi=(A, B)$ we have to perform the following steps. 
(1) Construct the involutive form of $A$.

(2) Prolong the system (if necessary) until the order of the system is higher than the the order of normal derivatives in the boundary operator.

(3) Construct an equivalent first-order system.

(4) Eliminate (if necessary) the extra variables using the algebraic relations in the system.

(5) Construct the compatibility operator for the normalised system with the formula (5.4).

(6) Construct the compatibility operator for the BV-operator using (5.5).

Next we will discuss how to implement these steps in practice.

\subsection{Constructions}

The first step is the Cartan-Kuranishi algorithm, and the second step is merely a simple differentiation. Since these steps are extensively discussed elsewhere, for example in [22], we will simply observe that they can be effectively performed, for example, with the DETools package in MuPAD.

Step (3): Equivalent first-order operator. This construction can be found in [19]. Let $A_{0}$ be a differential operator of order $q$ such that the first two steps of the above construction have already been performed, and let $A_{0}^{\prime}$ be an equivalent first-order operator. We want to write $A_{0}$ as $A_{0}=\bar{A} j^{q-1}$ where $\bar{A}$ is a first-order operator. We introduce new dependent variables for all derivatives of order less than or equal to $q-1$, and we denote them by $z^{j, \mu}$, with $|\mu| \leqslant q-1$. The operator $\bar{A}$ is obtained from $A$ by performing the following substitutions. (Obviously, there are many ways to perform such a substitution, as there are many ways to split the multi index $\mu$ into two parts. However, for our purposes any choice is fine.)

$$
y_{\mu}^{j} \longmapsto \begin{cases}z^{j, \mu}, & \text { if }|\mu| \leqslant q-1, \\ \partial^{\mu_{2}} z^{j, \mu_{1}}, & \text { if }|\mu|=q \text { where }\left|\mu_{1}\right|=q-1 \text { and } \mu_{1}+\mu_{2}=\mu .\end{cases}
$$

The desired first-order operator is $A_{0}^{\prime} z=\left(\bar{A} z, D^{q-1} z\right)$, where $\mathscr{D}^{q-1}$ is the compatibility operator for $j^{q-1}$. In coordinates it can be written as $\mathscr{D}^{q-1}=D_{1}^{q-1} \otimes I_{m}$, where $D_{1}^{q-1}$ is given by

$$
\partial_{i} z^{j, \mu}= \begin{cases}z^{j, \mu+\mathbf{1}_{i}}, & 0 \leqslant|\mu|<q-1,1 \leqslant i \leqslant n, \\ \partial_{k} z^{j, \mu-\mathbf{1}_{k}+\mathbf{1}_{i}}, & |\mu|=q-1, i>\operatorname{cls} \mu, k=\operatorname{cls} \mu .\end{cases}
$$

It is straightforward to verify that $A_{0}$ and $A_{0}^{\prime}$ are indeed equivalent, by considering the following diagram.

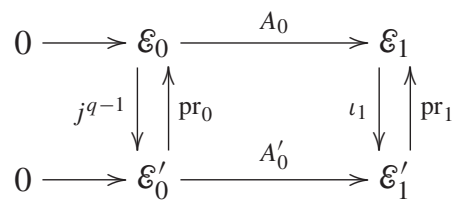

Since $\varepsilon_{0}^{\prime}$ is essentially $J_{q-1}\left(\varepsilon_{0}\right), \mathrm{pr}_{0}$ is simply the canonical projection, $\iota_{1}$ is the obvious injection and $\mathrm{pr}_{1}$ is the obvious projection. 
Step (4): Normalization. We have now constructed $A_{0}^{\prime}$, which is a first-order operator, equivalent to $A_{0}$. Also, the corresponding $B_{0}^{\prime}$ contains differentiations only in the directions tangent to the boundary. Now, algebraic relations in $A_{0}^{\prime}$ can be eliminated simply by performing Gaussian elimination followed by appropriate back-substitutions. We denote by $A_{0}^{\prime \prime}$ the resulting operator. Then we have the following diagram.

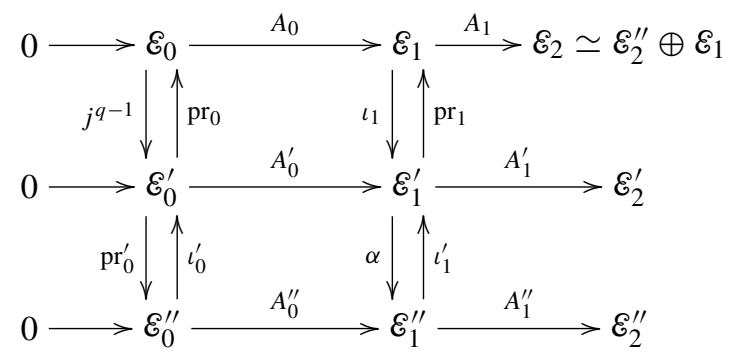

Here, the definitions of $\operatorname{pr}_{0}^{\prime}, \iota_{0}^{\prime}$ and $\iota_{1}^{\prime}$ are obvious, but $\alpha$, which describes the result of a Gaussian elimination, does not have any easy explicit expression.

However, we are really interested in BV-operators. This leads to the next diagram.

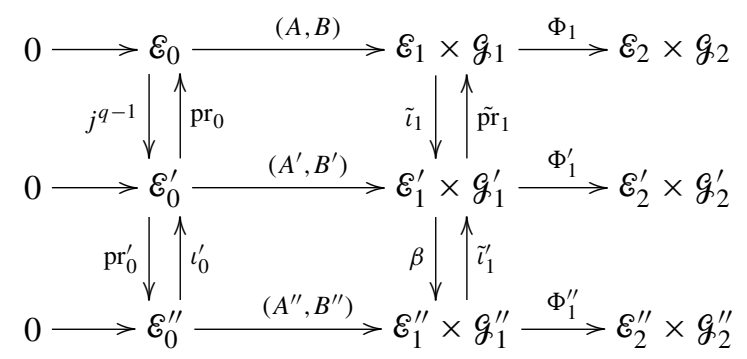

Let us briefly describe the various maps involved.

- The maps in the first column are the same as in the diagram (5.8).

- $\tilde{\iota}_{1}=\left(\iota_{1}\right.$, id $)$ where $\iota_{1}$ is as in (5.8). In particular, $g_{1}=g_{1}^{\prime}$.

- $\tilde{\mathrm{pr}}_{1}=\left(\mathrm{pr}_{1}\right.$, id) where $\mathrm{pr}_{1}$ is as in (5.8).

- $\tilde{\iota}_{1}^{\prime}=\left(\iota_{1}^{\prime}, \hat{\iota}_{1}\right)$ where $\iota_{1}^{\prime}$ is as in (5.8) and $\hat{\iota}_{1}$ is induced by $\iota_{0}^{\prime}$.

- The map $\beta$ is a DB operator, induced by the Gaussian elimination. We will write it as

$$
\beta\left(f^{\prime}, g^{\prime}\right)=\left(\alpha\left(f^{\prime}\right), \gamma \beta^{21}\left(f^{\prime}\right)+\beta^{22}\left(g^{\prime}\right)\right),
$$

where $\alpha$ is as in (5.8).

Step (5): Compatibility operator for the normalised BV-operator. Let $\Phi_{0}^{\prime \prime}=\left(A_{0}^{\prime \prime}, B_{0}^{\prime \prime}\right)$ be a normalised $\mathrm{BV}$-operator. We need to perform the following tasks.

5(a) Compute the tangent part of $A_{0}^{\prime \prime}$.

Let $M$ be the module generated by the rows of $A_{0}^{\prime \prime}$. We choose a monomial product ordering such that $\xi_{n}$ is bigger than all other $\xi_{i}$. Then we define a TOP module ordering using this monomial ordering, and compute the Gröbner basis of $M$. Now by Lemma $2.1 A_{0}^{\prime \prime \tau}$ is defined by the elements of the Gröbner basis that do not contain $\xi_{n}$. See Appendix A.5 for the appropriate computations with SINGULAR. 
5(b) Set $\Phi_{0}^{\prime \prime \tau}=\left(A_{0}^{\prime \prime \tau}, B_{0}^{\prime \prime}\right)$.

5(c) Compute $\Phi_{1}^{\prime \prime \tau}$, the compatibility operator of $\Phi_{0}^{\prime \prime \tau}$.

We choose a POT module ordering, and compute $\Phi_{1}^{\prime \prime \tau}$. Then Lemma 2.2 implies that we can now extract $\Upsilon^{\prime \prime} \tau$ by simple inspection.

5(d) The compatibility operator $\Phi_{1}^{\prime \prime}$ can now defined by the formula

$$
\Phi_{1}^{\prime \prime}\left(f^{\prime \prime}, g^{\prime \prime}\right)=\left(A_{1}^{\prime \prime} f^{\prime \prime}, \gamma \Phi_{1}^{21^{\prime \prime}} f^{\prime \prime}+\Phi_{1}^{22^{\prime \prime}} g^{\prime \prime}\right)=\left(A_{1}^{\prime \prime} f^{\prime \prime}, \Upsilon^{\prime \prime \tau}\left(\operatorname{pr}^{\tau} f^{\prime \prime}, g^{\prime \prime}\right)\right) \text {. }
$$

Step (6): Compatibility operator for the original involutive BV-operator. Using diagram (5.9), we may rewrite the formula (5.5) for the compatibility operator $\Phi_{1}$ of the operator $\Phi_{0}=(A, B)$ as

$$
\Phi_{1}=\left(\Phi_{1}^{\prime \prime} \beta \tilde{\iota}_{1}\right) \oplus\left(\mathrm{id}-\tilde{\mathrm{pr}}_{1} \tilde{\iota}_{1}^{\prime} \beta \tilde{\iota}_{1}-(A, B) \Psi_{0}\right)
$$

where $\Psi_{0}$ is determined by the equation

$$
\Psi_{0}(A, B)=\mathrm{id}-\operatorname{pr}_{0} \iota_{0}^{\prime} \mathrm{pr}_{0}^{\prime} j^{q-1} .
$$

If the system $A y=0$ does not contain algebraic relations between the dependent variables, then we may choose $\Psi_{0}=0$. If this is not the case, then we could as well apply Gaussian elimination to the original system and remove algebraic dependencies. Hence without loss of generality and for simplicity of notation we suppose in the sequel that $\Psi_{0}=0$. In this case we have

$$
\Phi_{1}^{11}=A_{1}=\left(A_{1}^{\prime \prime} \alpha \iota_{1}\right) \oplus\left(\mathrm{id}-\operatorname{pr}_{1} \iota_{1}^{\prime} \alpha \iota_{1}\right),
$$

where $A_{1}$ is the compatibility operator of $A$. The other parts of the compatibility operator are given by:

$$
\Phi_{1}^{21} f+\Phi_{1}^{22} g=\left(\Phi_{1}^{\prime \prime 21} \alpha \iota_{1}(f)+\Phi_{1}^{\prime \prime 22}\left(\gamma \beta^{21} \iota_{1}(f)+\beta^{22}(g)\right)\right) \oplus\left(g-\hat{\iota}_{1}\left(\gamma \beta^{21} \iota_{1}(f)+\beta^{22}(g)\right)\right) .
$$

We will see below that for the purposes of this paper we are particularly interested in $\Phi_{1}^{22}$. This is given by

$$
\Phi_{1}^{22}=\left(\Phi_{1}^{\prime \prime 22} \beta^{22}\right) \oplus\left(\mathrm{id}-\hat{\iota}_{1} \beta^{22}\right)
$$

Let us now consider an example of computation of the compatibility operator $A_{1}$ of the operator $A_{0}$ using the compatibility operator $A_{1}^{\prime \prime}$ of the equivalent normalised operator $A_{0}^{\prime \prime}$ and construction (5.5).

EXAmPLE 5.1. Consider the following familiar stationary Stokes problem in two dimensions:

$$
A:\left\{\begin{array}{l}
-\Delta u+\nabla p=0, \\
\nabla \cdot u=0,
\end{array} \quad \text { in } \mathbb{R}_{+}^{2}=\left\{x \in \mathbb{R}^{2}: x_{2}>0\right\},\right.
$$

where $u=\left(u^{1}, u^{2}\right)$ is the velocity field and $p$ is the pressure.

Completing it to the involutive form, we get the following overdetermined system:

$$
A_{0}:\left\{\begin{array}{l}
-\Delta u+\nabla p=0, \\
-\Delta p=0, \\
\nabla \cdot u=0, \\
u_{20}^{1}+u_{11}^{2}=0, \\
u_{11}^{1}+u_{02}^{2}=0,
\end{array} \quad \text { in } \mathbb{R}_{+}^{2} .\right.
$$


Let us go over to an equivalent normalised boundary value operator. Introducing nine new variables,

$$
\begin{aligned}
& z^{1,00}=u^{1} \\
& z^{2,00}=u^{2}, \quad z^{3,00}=p, \\
& z^{1,10}=u_{10}^{1} \text {, } \\
& z^{2,10}=u_{10}^{2} \text {, } \\
& z^{3,10}=p_{10}, \\
& z^{1,01}=u_{01}^{1}, \\
& z^{2,01}=u_{01}^{2}, \\
& z^{3,01}=p_{01},
\end{aligned}
$$

as in (5.6) and substituting them in (5.13), and also adding the compatibility equations (5.7), we get the first-order operator denoted by $A_{0}^{\prime}$. The corresponding system is not normalised since there is an algebraic relation $z^{1,10}+z^{2,01}=0$ between the dependent variables. Now, using this relation, we can exclude the variable $z^{2,01}$ from the system and obtain the normalised operator $A_{0}^{\prime \prime}$.

$$
A_{0}^{\prime}:\left\{\begin{array}{l}
-z_{10}^{1,10}-z_{01}^{1,01}+z^{3,10}=0 \\
-z_{10}^{2,10}-z_{01}^{2,01}+z^{3,01}=0 \\
-z_{10}^{3,10}-z_{01}^{3,01}=0 \\
z^{1,10}+z^{2,01}=0 \\
z_{10}^{1,10}+z_{10}^{2,01}=0 \\
z_{01}^{1,10}+z_{01}^{2,01}=0 \\
z_{10}^{1,00}-z^{1,10}=0 \\
z_{01}^{1,00}-z^{1,01}=0 \\
z_{10}^{2,00}-z^{2,10}=0 \\
z_{01}^{2,00}-z^{2,01}=0 \\
z_{10}^{3,00}-z^{3,10}=0 \\
z_{01}^{3,00}-z^{3,01}=0 \\
z_{01}^{1,10}-z_{10}^{1,01}=0 \\
z_{01}^{2,10}-z_{10}^{2,01}=0 \\
z_{01}^{3,10}-z_{10}^{3,01}=0
\end{array}\right.
$$$$
A_{0}^{\prime \prime}:\left\{\begin{array}{l}
-z_{10}^{1,10}+z^{3,10}-z_{01}^{1,01}=0 \\
z_{01}^{1,10}-z_{10}^{2,10}+z^{3,01}=0 \\
-z_{10}^{3,10}-z_{01}^{3,01}=0 \\
z_{10}^{1,00}-z^{1,10}=0 \\
z_{01}^{1,00}-z^{1,01}=0 \\
z_{10}^{2,00}-z^{2,10}=0 \\
z_{01}^{2,00}+z^{1,10}=0 \\
z_{10}^{3,00}-z^{3,10}=0 \\
z_{01}^{3,00}-z^{3,01}=0 \\
z_{01}^{1,10}-z_{10}^{1,01}=0 \\
z_{10}^{1,10}+z_{01}^{2,10}=0 \\
z_{01}^{3,10}-z_{10}^{3,01}=0
\end{array}\right.
$$

In this example we have the following fiber dimension of the bundles:

$$
\operatorname{dim}\left(\mathcal{E}_{1}\right)=6, \quad \operatorname{dim}\left(\mathcal{E}_{1}^{\prime}\right)=15, \quad \operatorname{dim}\left(\mathcal{E}_{1}^{\prime \prime}\right)=12 .
$$

To construct the compatibility operator $A_{1}^{\prime \prime}$ for the normalised operator $A_{0}^{\prime \prime}$ we compute with Singular the syzygy module generated by the rows of the full symbol matrix of the operator $A_{0}^{\prime \prime}$, and get

$$
\begin{aligned}
A_{1}^{\prime \prime}\left(f^{\prime \prime}\right)=( & -f_{01}^{\prime \prime 4}+f_{10}^{\prime \prime 5}-f^{\prime \prime 10}, f_{01}^{\prime \prime 6}+f_{10}^{\prime \prime 7}-f^{\prime \prime 11} \\
& \left.-f_{01}^{\prime \prime 8}+f_{10}^{\prime \prime 9}-f^{\prime \prime 12}, f_{10}^{\prime \prime 1}+f_{01}^{\prime \prime 2}+f^{\prime \prime 3}-f_{01}^{\prime \prime 10}+f_{10}^{\prime \prime 11}\right) .
\end{aligned}
$$

To construct the compatibility operator $A_{1}$ for the operator $A_{0}$, let us construct maps from the diagram (5.8). The map $\alpha: \mathcal{E}_{1}^{\prime} \rightarrow \mathcal{E}_{1}^{\prime \prime}$ is given by

$$
\alpha\left(f^{\prime}\right)=\left(f^{\prime 1}, f^{\prime 2}+f^{\prime 6}, f^{\prime 3}, f^{\prime 7}, f^{\prime 8}, f^{\prime 9}, f^{\prime 10}+f^{\prime 4}, f^{\prime 11}, f^{\prime 12}, f^{\prime 13}, f^{\prime 14}+f^{\prime 5}, f^{\prime 15}\right) .
$$

Combining this with the inclusion map $\iota_{1}(f)=(f, 0)$ gives

$$
\begin{gathered}
\alpha \iota_{1}(f)=\left(f^{1}, f^{2}+f^{6}, f^{3}, 0,0,0, f^{4}, 0,0,0, f^{5}, 0\right) ; \\
A_{1}^{\prime \prime} \alpha \iota_{1}(f)=\left(0, f_{10}^{4}-f^{5}, 0, f_{10}^{1}+f_{01}^{2}+f_{01}^{6}+f^{3}+f_{10}^{5}\right) .
\end{gathered}
$$




\section{The Shapiro-Lopatinskij condition}

The inclusion map $\iota_{1}^{\prime}: \mathcal{E}_{1}^{\prime \prime} \rightarrow \mathcal{E}_{1}^{\prime}$ is given by

$$
\iota_{1}^{\prime}\left(f^{\prime \prime}\right)=\left(f^{\prime \prime 1}, f^{\prime \prime 2}, f^{\prime \prime 3}, 0,0,0, f^{\prime \prime 4}, \ldots, f^{\prime \prime 12}\right) .
$$

So we have

$$
\iota_{1}^{\prime} \alpha \iota_{1}(f)=\left(f^{1}, f^{2}+f^{6}, f^{3}, 0,0,0,0,0,0, f^{4}, 0,0,0, f^{5}, 0\right) .
$$

The map $\operatorname{pr}_{1}: \mathcal{E}_{1}^{\prime} \rightarrow \mathcal{E}_{1}$ is defined by

$$
\begin{aligned}
\operatorname{pr}_{1}\left(f^{\prime}\right)=( & f^{\prime 1}-f_{10}^{\prime 7}-f_{01}^{\prime 8}+f^{\prime 11}, f^{\prime 2}-f_{10}^{\prime 9}-f_{01}^{\prime 10}+f^{\prime 12}, \\
& \left.f^{\prime 3}-f_{10}^{\prime 11}-f_{01}^{\prime 12}, f^{\prime 4}+f^{\prime 7}+f^{\prime 10}, f^{\prime 5}+f_{10}^{\prime 7}+f_{10}^{\prime 10}, f^{\prime 6}+f_{01}^{\prime 7}+f_{01}^{\prime 10}\right) .
\end{aligned}
$$

Hence we get

$$
\left(\mathrm{id}-\mathrm{pr}_{1} \iota_{1}^{\prime} \alpha \iota_{1}\right)(f)=\left(0, f_{01}^{4}-f^{6}, 0,0, f^{5}-f_{10}^{4}, f^{6}-f_{01}^{4}\right) .
$$

Thus, (5.5) gives the following compatibility operator $A_{1}$ of $A_{0}$ :

$$
A_{1}(f)=\left(f_{01}^{4}-f^{6}, f_{10}^{4}-f^{5}, f_{10}^{1}+f_{01}^{2}+f^{3}+f_{10}^{5}+f_{01}^{6}\right) .
$$

Note that we can also compute the compatibility operator $A_{1}$ of the operator $A_{0}$ by computing with SingULAR the syzygy module of the module generated by the rows of the full symbol matrix of $A_{0}$. However, the above computations are needed when we consider the Stokes problem with boundary conditions in the next example. In particular, we show how to compute the part $\Phi_{1}^{22}$ of the compatibility operator $\Phi_{1}$ for $\Phi_{0}=(A, B)$, since that is necessary for checking the SL-condition for overdetermined boundary problems.

EXAmPLE 5.2. Let us consider the operator $A_{0}$ given by (5.13). Then the tangent part of the equivalent normalised operator operator $A_{0}^{\prime \prime}$ is

$$
A_{0}^{\prime \prime \tau} z=\left(z_{10}^{2,10}-z_{10}^{1,01}-z^{3,01},-z^{3,10}+z_{10}^{3,00},-z^{2,10}+z_{10}^{2,00},-z^{1,10}+z_{10}^{1,00}\right) .
$$

Let us define the following boundary operator

$$
B:\left\{\begin{array}{l}
u^{1}+u_{01}^{2}-p_{10}=0, \\
-p-2 u_{10}^{2}+p_{10}=0, \\
u_{10}^{1}-u_{10}^{2}=0, \\
-u_{10}^{2}+p_{10}=0, \\
\nabla \cdot u=0
\end{array} \quad \text { on } \Gamma=\partial \mathbb{R}_{+}^{2},\right.
$$

for the operator $A_{0}$. Then we get

$$
B^{\prime}:\left\{\begin{array}{l}
z^{1,00}+z^{2,01}-z^{3,10}=0, \\
-z^{3,00}-2 z^{2,10}+z^{3,10}=0, \\
z^{1,10}-z^{2,10}=0, \\
-z^{2,10}+z^{3,10}=0, \\
z^{1,10}+z^{2,01}=0 ;
\end{array} \quad B^{\prime \prime}:\left\{\begin{array}{l}
z^{1,00}-z^{1,10}-z^{3,10}=0, \\
-z^{3,00}-2 z^{2,10}+z^{3,10}=0, \\
z^{1,10}-z^{2,10}=0, \\
-z^{2,10}+z^{3,10}=0 .
\end{array}\right.\right.
$$

In this example we have the following fiber dimension of the bundles:

$$
\operatorname{dim}\left(g_{1}\right)=5, \quad \operatorname{dim}\left(g_{1}^{\prime}\right)=5, \quad \operatorname{dim}\left(g_{1}^{\prime \prime}\right)=4, \quad \operatorname{dim}\left(\mathscr{E}_{1}^{\prime \prime \tau}\right)=4
$$

Let us compute the compatibility operator $\Phi_{1}^{\prime \prime \tau}$ for the operator $\Phi^{\prime \prime} \tau z=\left(A_{0}^{\prime \prime \tau} z, B^{\prime \prime} z\right)$ defined on $\Gamma$. Computing with Singular the syzygy module for the module generated by the rows 


\section{The Shapiro-Lopatinskij condition}

of the full symbol matrix of the operator $\Phi^{\prime \prime \tau}$, we have

$$
\left(0,-2 \xi_{1}+1,0,-\xi_{1}-1, \xi_{1}^{2}+\xi_{1},-2 \xi_{1}^{2}+\xi_{1}, \xi_{1}^{2}-1,3 \xi_{1}^{2}-2 \xi_{1}+1\right) .
$$

In this example we find that the compatibility operator $\Phi_{1}^{\prime \prime \tau}$ is in fact $\Upsilon^{\tau}$, and we have

$$
\begin{aligned}
\Phi_{1}^{\prime \prime \tau}\left(f^{\tau}, g^{\prime \prime}\right)= & -2 f_{10}^{2, \tau}+f^{2, \tau}-f_{10}^{4, \tau}-f^{4, \tau} \\
& +g_{20}^{\prime \prime 1}+g_{10}^{\prime \prime 1}-2 g_{20}^{\prime \prime 2}+g_{10}^{\prime \prime 2}+g_{20}^{\prime \prime 3}-g^{\prime \prime 3}+3 g_{20}^{\prime \prime 4}-2 g_{10}^{\prime \prime 4}+g^{\prime \prime 4} .
\end{aligned}
$$

The projection $\mathrm{pr}^{\tau}: \mathcal{E}_{1}^{\prime \prime} \rightarrow \mathcal{E}_{1}^{\prime \prime \tau}$ is given by

$$
\operatorname{pr}^{\tau}\left(f^{\prime \prime}\right)=\left(\left.f^{\prime \prime 10}\right|_{\Gamma}-\left.f^{\prime \prime 2}\right|_{\Gamma},\left.f^{\prime \prime 8}\right|_{\Gamma},\left.f^{\prime \prime 6}\right|_{\Gamma},\left.f^{\prime \prime 4}\right|_{\Gamma}\right) .
$$

Thus the compatibility operator $\Phi_{1}^{\prime \prime}$ for the normalised operator $\left(A_{0}^{\prime \prime}, B^{\prime \prime}\right)$ is

$$
\begin{aligned}
\Phi_{1}^{\prime \prime}\left(f^{\prime \prime}, g^{\prime \prime}\right)= & \left(A_{1}^{\prime \prime} f^{\prime \prime},-\left.2 f_{10}^{\prime \prime 8}\right|_{\Gamma}+\left.f^{\prime \prime 8}\right|_{\Gamma}-\left.f_{10}^{\prime \prime 4}\right|_{\Gamma}-\left.f^{\prime \prime 4}\right|_{\Gamma}+\right. \\
& \left.g_{20}^{\prime \prime 1}+g_{10}^{\prime \prime 1}-2 g_{20}^{\prime \prime 2}+g_{10}^{\prime \prime 2}+g_{20}^{\prime \prime 3}-g^{\prime \prime 3}+3 g_{20}^{\prime \prime 4}-2 g_{10}^{\prime \prime 4}+g^{\prime \prime 4}\right),
\end{aligned}
$$

which implies that

$$
\Phi_{1}^{\prime \prime 22}\left(g^{\prime \prime}\right)=g_{20}^{\prime \prime 1}+g_{10}^{\prime \prime 1}-2 g_{20}^{\prime \prime 2}+g_{10}^{\prime \prime 2}+g_{20}^{\prime \prime 3}-g^{\prime \prime 3}+3 g_{20}^{\prime \prime 4}-2 g_{10}^{\prime \prime 4}+g^{\prime \prime 4} \text {. }
$$

The $\operatorname{map} \beta: \varepsilon_{1}^{\prime} \times g_{1} \rightarrow \mathcal{E}_{1}^{\prime \prime} \times \mathcal{g}_{1}^{\prime \prime}$ is defined by

$$
\beta\left(f^{\prime}, g^{\prime}\right)=\left(\alpha\left(f^{\prime}\right), g^{\prime 1}-\left.f^{\prime 4}\right|_{\Gamma}, g^{\prime 2}, g^{\prime 3}, g^{\prime 4}\right) \text {. }
$$

Hence we obtain

$$
\beta^{22}\left(g^{\prime}\right)=\left(g^{\prime 1}, g^{\prime 2}, g^{\prime 3}, g^{\prime 4}\right) .
$$

So the formula (5.11) yields

$$
\Phi_{1}^{22} g=\left(g_{20}^{1}+g_{10}^{1}-2 g_{20}^{2}+g_{10}^{2}+g_{20}^{3}-g^{3}+3 g_{20}^{4}-2 g_{10}^{4}+g^{4}, 0,0,0,0, g^{5}\right) .
$$

\section{Overdetermined elliptic boundary problems (the SL-condition)}

\subsection{Well-posed problems for overdermined elliptic PDES}

From now on, let $\mathrm{B}_{w}$ be the weighted principal symbol of $B=\left(B_{1}, \ldots, B_{\tilde{v}}\right)$ with weights $t_{j}=0$ for the dependent variables and $r_{i}$ for the equations, where $r_{i}$ is the order of the operator $B_{i}, i=1, \ldots, \tilde{v}$. Let $\Phi_{1}$ be a compatibility operator for $(A, B)$. We denote by $\left(\Phi_{1}^{22}\right)_{w}$ the weighted principal symbol of $\Phi_{1}^{22}$ with the weights $-r_{j}$ for dependent variables and the weights $\delta_{i}$ for equations. We denote by $\tau$ the number of rows of $\Phi_{1}^{22}$.

Set $r=\left(r_{1}, \ldots, r_{\tilde{v}}\right)$ and $\delta=\left(\delta_{1}, \ldots, \delta_{\tau}\right)$, and let

$$
g_{1}=\bigoplus_{\ell=1}^{\tilde{v}} G_{1}^{\ell} \quad \text { and } \quad g_{2}=\bigoplus_{\ell=1}^{\tau} G_{2}^{\ell}
$$

be the direct sum decompositions of the bundles $g_{1}$ and $g_{2}$.

Definition 6.1. An operator $(A, B)$ satisfies the $S L$-condition if, for any $x \in \Gamma$ and $\xi^{\prime} \neq 0$, the complex

$$
\left.\left.0 \longrightarrow \operatorname{ker}\left(\mathrm{A}\left(x, \xi^{\prime}, D_{n}\right)\right) \cap \mathfrak{M}_{+} \stackrel{\mathrm{B}_{w}\left(x, \xi^{\prime}, D_{n}\right)}{\longrightarrow} g_{1}\right|_{x} \stackrel{\left(\Phi_{1}^{22}\right)_{w}\left(x, \xi^{\prime}\right)}{\longrightarrow} g_{2}\right|_{x}
$$

is exact.

Here the operator $\mathrm{B}_{w}\left(x, \xi^{\prime}, D_{n}\right)$ is interpreted as $\left.\mathrm{B}_{w}\left(x, \xi^{\prime}, D_{n}\right) u\left(x_{n}\right)\right|_{x_{n}=0}$. 
REMARK 6.1. Perhaps it would be more appropriate to use the term the generalized SLcondition in the above definition. However, for simplicity of language we prefer to use 'the SL-condition', even in this case.

The basic theorem on the solvability of the boundary problem (5.1) is as follows.

TheOREM 6.1 (see [9]). Let $(A, B): C^{\infty}\left(\varepsilon_{0}\right) \rightarrow C^{\infty}\left(\varepsilon_{1}\right) \times C^{\infty}\left(\mathcal{g}_{1}\right)$ be a regular boundary value problem operator, and suppose that $A$ is involutive and elliptic, $(A, B)$ satisfies the SL-condition and the number $\operatorname{dim}\left(\operatorname{ker}\left(\mathrm{A}\left(x, \xi^{\prime}, D_{n}\right)\right)\right)$ does not depend on $\left(x, \xi^{\prime}\right)$. In this case, if all the mappings in the complex

$$
0 \longrightarrow H_{s}\left(\mathcal{E}_{0}\right) \stackrel{(A, B)}{\longrightarrow} H_{s-q}\left(\mathcal{E}_{1}\right) \times H_{s-r-1 / 2}\left(g_{1}\right) \stackrel{\Phi_{1}}{\longrightarrow} H_{s-q-1}\left(\mathcal{E}_{2}\right) \times H_{s-\eta}\left(\mathscr{g}_{2}\right),
$$

where $\eta$ is some appropriate vector, are bounded, then there is a number such that its cohomologies are finite-dimensional and their dimensions remain invariant when $s$ is replaced by $s^{\prime}>s$.

We would like to propose a computational test for checking the SL-condition, but first we need to consider some preliminary results.

\subsection{Reduction of an overdetermined system to an equivalent square upper triangular one}

Fixing some boundary point $\left(x^{\prime}, 0\right) \in \Gamma$ and some vector $\xi^{\prime} \neq 0$, we consider an overdetermined ordinary differential system with constant coefficients

$$
\mathrm{A}\left(\xi^{\prime}, D_{n}\right) u\left(x_{n}\right)=0 .
$$

We will first reduce this system to the upper triangular system

$$
\mathrm{U}\left(\xi^{\prime}, D_{n}\right) u\left(x_{n}\right)=\left(\begin{array}{cccc}
* & * & \ldots & * \\
& * & \ldots & * \\
& & \ddots & \vdots \\
& & & * \\
& & & \vdots \\
& & & *
\end{array}\right) u\left(x_{n}\right)=0
$$

where $\bigcup_{i j}=0$ if $i>j$ and $j<m$. Writing now $\zeta$ for $D_{n}$, we may interpret the elements of $A$ and $U$ as elements of the ring $\mathbb{R}[\zeta]$.

THeOREM 6.2. There is a matrix $T \in(\mathbb{R}[\zeta])^{k \times k}$ such that $T \mathrm{~A}=\mathrm{U}$ with $\mathrm{U}$ as in (6.3) and $\operatorname{det}(T)=1$.

Proof. Let $a$ and $b$ be two rows of A, and let $C$ be a $2 \times m$ matrix whose first row is $a$ and whose second row is $b$. Now we want to construct a matrix $P$ such that $P C$ has zero in position $(2,1)$ and $\operatorname{det}(P)=1$.

First note that if $b_{1}=0$, then we take $P=I$ and if $a_{1}=0$, then

$$
P=\left(\begin{array}{cc}
0 & -1 \\
1 & 0
\end{array}\right) \text {. }
$$

Now let us suppose that $a_{1} \neq 0$ and $b_{1} \neq 0$. Let $h=\operatorname{gcd}\left(a_{1}, b_{1}\right)$ be the greatest common divisor of $a_{1}$ and $b_{1}$. Then we have $a_{1}=-h p_{22}$ and $b_{1}=h p_{21}$ for some polynomials 
$p_{21}$ and $p_{22}$ such that $\operatorname{gcd}\left(p_{21}, p_{22}\right)=1$. Denote by $\left\langle p_{21}, p_{22}\right\rangle$ the ideal generated by the polynomials $p_{21}$ and $p_{22}$. Since

$$
\left\langle p_{21}, p_{22}\right\rangle=\left\langle\operatorname{gcd}\left(p_{21}, p_{22}\right)\right\rangle=\langle 1\rangle=\mathbb{R}[\zeta],
$$

there are polynomials $p_{11}$ and $p_{12}$ such that $\operatorname{det}\left(P_{1}\right)=1$, where

$$
P=\left(\begin{array}{ll}
p_{11} & p_{12} \\
p_{21} & p_{22}
\end{array}\right)
$$

Then by construction we have

$$
P C=\left(\begin{array}{llll}
* & * & \ldots & * \\
0 & * & \ldots & *
\end{array}\right),
$$

where $*$ denotes some element.

Now consider the following matrix:

$$
\tilde{P}=\left(\begin{array}{ccccc}
I_{s_{1}} & 0 & 0 & 0 & 0 \\
0 & p_{11} & 0 & p_{12} & 0 \\
0 & 0 & I_{s_{2}} & 0 & 0 \\
0 & p_{21} & 0 & p_{22} & 0 \\
0 & 0 & 0 & 0 & I_{s_{3}}
\end{array}\right),
$$

where $I_{i}$ is the identity $i \times i$ matrix and $k=s_{1}+s_{2}+s_{3}+2$ (if $s_{i}=0$, then we delete the corresponding row and column). Now, if we construct polynomials $p_{i j}$ as above, then $\operatorname{det}(\tilde{P})=1$ and multiplying A by $\tilde{P}_{1}$ produces a zero in the row $s_{1}+s_{2}+2$.

Then choosing appropriate matrices of this form, we can form a product $T=\tilde{P}_{j} \ldots \tilde{P}_{1}$ which has the properties stated in the theorem.

Let us define an $m \times m$ matrix $\tilde{U}$ by $\tilde{U}_{i j}=\bigcup_{i j}$, except that $\tilde{U}_{m m}=\operatorname{gcd}\left(\bigcup_{m m}, \ldots, \bigcup_{k m}\right)$.

Substituting now $D_{n}$ for $\zeta$, we consider the upper triangular square system of ordinary differential equations

$$
\tilde{\mathrm{U}}\left(\xi^{\prime}, D_{n}\right) u\left(x_{n}\right)=0, \quad x_{n}>0 \text {. }
$$

LemMa 6.1. For any fixed $\xi^{\prime} \neq 0$, the solution spaces of the systems (6.2) and (6.3) are equal. In particular, for any fixed $\xi^{\prime} \neq 0$,

$$
\operatorname{dim}\left(\operatorname{ker}\left(\mathrm{A}\left(x, \xi^{\prime}, D_{n}\right)\right) \cap \mathfrak{M}_{+}\right)=\operatorname{dim}\left(\operatorname{ker}\left(\tilde{U}\left(x, \xi^{\prime}, D_{n}\right)\right) \cap \mathfrak{M}_{+}\right)=v .
$$

Proof. The statement of this lemma follows from the equivalence of systems (6.6) and (6.2), and Lemma 4.1.

\subsection{Algebraic criterion for checking the SL-condition}

First we fix some $\xi^{\prime} \neq 0$. Let $\zeta_{1}, \ldots, \zeta_{\nu}$ be the roots of the polynomial $p_{\mathrm{A}} \in \mathbb{R}[\zeta]$ lying in the upper half of the complex plane. We set

$$
p_{\mathrm{A}}^{+}=\left(\zeta-\zeta_{1}\right) \ldots\left(\zeta-\zeta_{\nu}\right)=\sum_{j=0}^{\nu} b_{j} \zeta^{j}
$$

and introduce the polynomials $p_{l}^{+}, l=1, \ldots, v$, as in (2.2). 
Lemma 6.2. Let $\mathrm{A}_{s}$ be some $m \times m$ submatrix of the matrix $\mathrm{A}$. Then the columns of the matrix

$$
W_{l}\left(x_{n}\right)=\frac{1}{2 \pi i} \oint_{\gamma_{+}} \frac{\operatorname{adj}\left(\mathrm{A}_{s}(\zeta)\right) p_{l}^{+}(\zeta) e^{i \zeta x_{n}}}{p_{\mathrm{A}}^{+}(\zeta)} d \zeta, \quad l=1, \ldots, v,
$$

belong to the space $\operatorname{ker}\left(\mathrm{A}\left(D_{n}\right)\right) \cap \mathfrak{M}_{+}$.

Proof. Note that

$$
\mathrm{A}\left(D_{n}\right) W_{l}\left(x_{n}\right)=\frac{1}{2 \pi i} \oint_{\gamma_{+}} \frac{\mathrm{A}(\zeta) \operatorname{adj}\left(\mathrm{A}_{s}(\zeta)\right) p_{l}^{+}(\zeta) e^{i \zeta x_{n}}}{p_{\mathrm{A}}^{+}(\zeta)} d \zeta, \quad l=1, \ldots, v
$$

Hence, if we show that all elements of the matrix $\tilde{A}=\operatorname{A} \operatorname{adj}\left(\mathrm{A}_{s}\right)$ are divisible by the polynomial $p_{\mathrm{A}}^{+}$, then by the Cauchy integral theorem we get $\mathrm{A}\left(D_{n}\right) W_{l}\left(x_{n}\right)=0$. Since $\Re(i \zeta)<0$, we see that the columns of $W_{l}$ belong to $\operatorname{ker}\left(\mathrm{A}\left(D_{n}\right)\right) \cap \mathfrak{M}_{+}$.

Let us now show that elements of $\tilde{\mathrm{A}}$ are divisible by $p_{\mathrm{A}}^{+}$. Let us denote by $\left(a_{i 1}, \ldots, a_{i m}\right)$ the $i$ th row of the matrix A. Then, by the definition of the adjoint matrix for the $i$ th row of $\tilde{\mathrm{A}}$, we get

$$
\tilde{\mathrm{A}}_{i i}=\operatorname{det}\left(\mathrm{A}_{s}\right), \quad \tilde{\mathrm{A}}_{i j}=0, \quad i \neq j,
$$

if the $i$ th row of the matrix A belongs to $\mathrm{A}_{s}$, or

$$
\tilde{\mathrm{A}}_{i}=\left(a_{i 1}, \ldots, a_{i m}\right) \operatorname{adj}\left(\mathrm{A}_{s}\right),
$$

otherwise. In case of the first possibility, by the definition of the polynomial $p_{\mathrm{A}}^{+}$every $\tilde{\mathrm{A}}_{i j}$ is divisible by $p_{\mathrm{A}}^{+}$.

Let us now consider the second case. By the definition of the adjoint matrix, the elements of $\operatorname{adj}\left(\mathrm{A}_{s}\right)$ are $\left(\operatorname{adj}\left(\mathrm{A}_{s}\right)\right)_{i j}=(-1)^{i+j} B_{j i}$, where $B_{i j}$ is the $(i, j)$ minor of the matrix $\mathrm{A}_{s}$. Hence

$$
\tilde{\mathrm{A}}_{i j}=a_{i_{1}}(-1)^{1+j} B_{j 1}+\ldots+a_{i_{m}}(-1)^{m+j} B_{j m} .
$$

But then $\tilde{\mathrm{A}}_{i j}$ is \pm the determinant of the $m \times m$ submatrix of $\mathrm{A}$ which consists of the row $\left(a_{i 1}, \ldots, a_{i m}\right)$ and all rows of $\mathrm{A}_{s}$ except the $j$ th row. So, again by the definition of the polynomial $p_{\mathrm{A}}^{+}$, every $\tilde{\mathrm{A}}_{i j}$ is divisible by $p_{\mathrm{A}}^{+}$.

LEMma 6.3. There is a $m \times m$ submatrix of A such that some element of its adjoint is not divisible by $p_{\mathrm{A}}^{+}$.

Proof. Suppose the contrary - that is, that all elements of all submatrices $\operatorname{adj}\left(\mathrm{A}_{S}\right)$ are divisible by $p_{\mathrm{A}}^{+}: \operatorname{adj}\left(\mathrm{A}_{s}\right)=p_{\mathrm{A}}^{+} C_{s}$ for all $s=1, \ldots, r$. Setting $q_{s}=\operatorname{det}\left(\mathrm{A}_{s}\right)$ and by the property of adjoint matrix we get $\operatorname{det}\left(\operatorname{adj}\left(\mathrm{A}_{s}\right)\right)=q_{s}^{m-1}$. So we have $\left(p_{\mathrm{A}}^{+}\right)^{m} \operatorname{det}\left(C_{s}\right)=q_{s}^{m-1}$. Since $p_{\mathrm{A}}=\operatorname{gcd}\left(q_{1}, \ldots, q_{r}\right)$, we get $q_{s}=p_{\mathrm{A}}^{+} \tilde{q}_{s}$ for all $s=1, \ldots, r$. Thus, $p_{\mathrm{A}}^{+} \operatorname{det}\left(C_{s}\right)=$ $\left(\tilde{q}_{s}\right)^{m-1}$. Hence, $\tilde{q}_{s}, s=1, \ldots, r$, are divisible by $p_{\mathrm{A}}^{+}$, and so $p_{\mathrm{A}}$ is divisible by $\left(p_{\mathrm{A}}^{+}\right)^{2}$. But this contradicts the definition of $p_{\mathrm{A}}^{+}$.

Let $\mathrm{A}_{s}$ be as in Lemma 6.3, and denote by $v$ some column of the matrix $\operatorname{adj}\left(\mathrm{A}_{s}\right)$ which is nonzero modulo $p_{\mathrm{A}}^{+}$. Also, as in the square case, we set

$$
\omega^{l}\left(x_{n}\right)=\frac{1}{2 \pi i} \oint_{\gamma_{+}} \frac{v(\zeta) p_{l}^{+}(\zeta) e^{i \zeta x_{n}}}{p_{\mathrm{A}}^{+}(\zeta)} d \zeta, \quad l=1, \ldots, v,
$$

and we let $\omega$ be a matrix with columns $\omega^{l}$. 
LEMma 6.4. The columns of $\omega$ are a basis of the space $\operatorname{ker}\left(\mathrm{A}\left(D_{n}\right)\right) \cap \mathfrak{M}_{+}$.

Proof. Due to Lemma 6.2 and Lemma 6.3, the proof is the same as in the square case (see Lemma 4.3).

Let us now consider the vector $h=\mathrm{B}_{w} v$. Dividing each element of $h$ by the polynomial $p_{\mathrm{A}}^{+}$, we get

$$
h=q p_{\mathrm{A}}^{+}+\mathrm{h}, \quad \text { where } \mathrm{h}=\sum_{\tau=0}^{\nu-1} \mathrm{~h}^{\tau} \zeta^{\tau} .
$$

Let us introduce the $\tilde{v} \times v$ matrix $\mathscr{H}$ :

$$
\mathscr{H}=\left(\mathrm{h}^{0}, \ldots, \mathrm{h}^{\nu-1}\right) .
$$

Now we formulate the algebraic criterion for checking the coerciveness condition.

THEOREM 6.3. The following statements are equivalent.

(i) An operator $(A, B)$ satisfies the SL-condition.

(ii) For any $x \in \Gamma$ and $\xi^{\prime} \neq 0$, there is a column $v$ of some matrix $\operatorname{adj}\left(\mathrm{A}_{S}\right)$ that is not divisible by $p_{\mathrm{A}}^{+}$such that $\operatorname{rank}(\mathcal{H}, d)=v$ for all $d \in \operatorname{ker}\left(\left(\Phi_{1}^{22}\right)_{w}\right)$ and $\operatorname{dim}\left(\operatorname{ker}\left(\left(\Phi_{1}^{22}\right)_{w}\right)\right)=v$, where $\mathscr{H}$ is defined as in (6.7) using $v$.

Proof. (ii) $\Longrightarrow$ (i): First we fix some $x \in \Gamma$ and $\xi^{\prime} \neq 0$. We take a column $v$ of some matrix $\operatorname{adj}\left(\mathrm{A}_{S}\right)$ that is not divisible by $p_{\mathrm{A}}^{+} \operatorname{such}$ that $\operatorname{rank}(\mathscr{H}, d)=v$ for all $d \in \operatorname{ker}\left(\left(\Phi_{1}^{22}\right)_{w}\right)$. Since $\left(\Phi_{1}^{22}\right)_{w}$ is a linear operator, we get $0 \in \operatorname{ker}\left(\left(\Phi_{1}^{22}\right)_{w}\right)$. This implies that $\operatorname{rank}(\mathscr{H})=v$. So for all $d \in \operatorname{ker}\left(\left(\Phi_{1}^{22}\right)_{w}\right)$ we have $\operatorname{rank}(\mathscr{H})=\operatorname{rank}(\mathscr{H}, d)$ and therefore the system $\mathscr{H} c=d$ has a solution. Using this vector $c$ we construct the following function $u\left(x_{n}\right)=\omega\left(x_{n}\right) c$. Lemma 6.4 implies that $u \in \operatorname{ker}\left(\mathrm{A}\left(D_{n}\right)\right) \cap \mathfrak{M}_{+}$. By Lemma 4.4 we get $\left.\mathrm{B}_{w}\left(D_{n}\right) u\left(x_{n}\right)\right|_{x_{n}=0}=\mathscr{H} c=$ $d$. So we have $\operatorname{ker}\left(\left(\Phi_{1}^{22}\right)_{w}\right) \subset \operatorname{im}\left(\mathrm{B}_{w}\right)$. The definition of a compatibility operator implies that $\operatorname{im}\left(\mathrm{B}_{w}\right) \subset \operatorname{ker}\left(\left(\Phi_{1}^{22}\right)_{w}\right)$. Hence, $\operatorname{ker}\left(\left(\Phi_{1}^{22}\right)_{w}\right)=\operatorname{im}\left(\mathrm{B}_{w}\right)$. So $\operatorname{dim}\left(\operatorname{im}\left(\mathrm{B}_{w}\right)\right)=v$. By Lemma 6.1 we know that $\operatorname{dim}\left(\operatorname{ker}\left(\mathrm{A}\left(D_{n}\right)\right) \cap \mathfrak{M}_{+}\right)=v$. Since $\mathrm{B}_{w}$ is a linear operator, we get $\operatorname{dim}\left(\operatorname{ker}\left(\mathrm{B}_{w}\right)\right)=0$. So $\operatorname{ker}\left(\mathrm{B}_{w}\right)=\{0\}$, and hence the complex (6.1) is exact.

(i) $\Longrightarrow$ (ii): Suppose now that the complex (6.1) is exact. Take some $x \in \Gamma$ and $\xi^{\prime} \neq 0$. This, together with the linearity of the operator $\mathrm{B}_{w}$, implies that $\operatorname{dim}\left(\operatorname{im}\left(\mathrm{B}_{w}\right)\right)=$ $\operatorname{dim}\left(\operatorname{ker}\left(\left(\Phi_{1}^{22}\right)_{w}\right)\right)=v$. Take some column $v$ of some $\operatorname{adj}\left(\mathrm{A}_{s}\right)$ that is not divisible by $p_{\mathrm{A}}^{+}$ and construct the matrix $\omega$ using $v$. According to Lemma 6.4 the columns of the matrix $\omega$ are a basis of the space $\operatorname{ker}\left(\mathrm{A}\left(D_{n}\right)\right) \cap \mathfrak{M}_{+}$. Since $\mathrm{B}_{w}$ is a linear operator, $\operatorname{ker}\left(\mathrm{B}_{w}\right)=\{0\}$ and $\operatorname{dim}\left(\operatorname{im}\left(\mathrm{B}_{w}\right)\right)=v$, we find that the columns of $\left.\mathrm{B}_{w} \omega\left(x_{n}\right)\right|_{x_{n}=0}=\mathscr{H}$ are a basis of the space $\operatorname{im}\left(\mathrm{B}_{w}\right)$. We see also that $\operatorname{ker}\left(\left(\Phi_{1}^{22}\right)_{w}\right)=\operatorname{im}\left(\mathrm{B}_{w}\right)$, and hence $\operatorname{rank}(\mathcal{H}, d)=v$ for all $d \in \operatorname{ker}\left(\left(\Phi_{1}^{22}\right)_{w}\right)$.

REMARK 6.2. If condition (ii) of Theorem 6.3 holds, then for any column of any matrix $\operatorname{adj}\left(\mathrm{A}_{1}\right), \ldots, \operatorname{adj}\left(\mathrm{A}_{r}\right)$ that is not divisible by $p_{\mathrm{A}}^{+}$, we have $\operatorname{rank}(\mathscr{H}, d)=v$ for all $d \in$ $\operatorname{ker}\left(\left(\Phi_{1}^{22}\right)_{w}\right)$, where $\mathscr{H}$ is defined using this column.

6.4. Computational test for checking the SL-condition in the case of two independent variables

Let $\hat{p}_{\mathrm{A}} \in \mathbb{K}[\zeta]$, where $\mathbb{K}$ is, as before, the dehomogenised characteristic polynomial, and let us denote by $\hat{\mathrm{A}}, \hat{\mathrm{B}}_{w}$ and $\left(\hat{\Phi}_{1}^{22}\right)_{w}$ the dehomogenised symbol matrices. Also, we denote 
the splitting field of $\hat{p}_{\mathrm{A}}$ by $\mathbb{K}(\alpha)$. It is evident that $\hat{p}_{\mathrm{A}}=\operatorname{gcd}\left(\operatorname{det}\left(\hat{\mathrm{A}}_{1}\right), \ldots, \operatorname{det}\left(\hat{\mathrm{A}}_{r}\right)\right)$ and we have homomorphisms $\iota$ and $\tilde{\imath}$ as in (4.7) and (4.8).

We define polynomials $\hat{p}_{\mathrm{A}}^{+}, \tilde{p}_{\mathrm{A}}^{+}$and $\tilde{p}_{\mathrm{A}}^{-}$as in (4.9) and (4.10). As in the square case, we will work with the polynomial $\hat{p}_{A}^{+}$in our computational test. The following result is similar to Lemma 4.5.

Lemma 6.5. There is an element $\hat{d}$ of some $\operatorname{adj}\left(\hat{\mathrm{A}}_{s}\right)$ such that:

(i) $\hat{d}$ is not divisible by $\hat{p}_{\mathrm{A}}^{+}$;

(ii) $\hat{d}$ is not divisible by $\tilde{\imath}\left(\hat{p}_{\mathrm{A}}^{+}\right)$;

(iii) the corresponding element $d$ of $\operatorname{adj}\left(\mathrm{A}_{s}\right)$ is not divisible by $\tilde{p}_{\mathrm{A}}^{+}$;

(iv) for any fixed $\xi_{1} \neq 0$ the corresponding element $d$ of $\operatorname{adj}\left(\mathrm{A}_{s}\right)$ is not divisible by $p_{\mathrm{A}}^{+}$.

Suppose that $\hat{d}$ is in the $j$ th column of $\operatorname{adj}\left(\hat{\mathrm{A}}_{s}\right)$. We denote by $\hat{v}$ the $j$ th column of $\operatorname{adj}\left(\hat{\mathrm{A}}_{s}\right)$, and by $v$ the $j$ th column of $\operatorname{adj}\left(\mathrm{A}_{s}\right)$.

Now, for any fixed $\xi_{1} \neq 0$, we construct the matrix $\mathscr{H}$ as in (6.7) using the $j$ th column of $\operatorname{adj}\left(\mathrm{A}_{s}\right)$ and polynomial $p_{\mathrm{A}}^{+}$. Then, working with polynomials in two variables in the same way, we construct a matrix $\tilde{\mathscr{H}}$ using the same $j$ th column of $\operatorname{adj}\left(\mathrm{A}_{s}\right)$ and polynomial $\tilde{p}_{\mathrm{A}}^{+}$. It is immediate that for any fixed $\xi_{1} \neq 0$,

$$
\mathscr{H}= \begin{cases}\tilde{\mathscr{H}}, & \text { if } \xi_{1}>0, \\ \tilde{\mathscr{H}}, & \text { if } \xi_{1}<0 .\end{cases}
$$

Let us now consider dehomogenised symbols and construct a matrix $\hat{\mathscr{H}}$ using the $j$ th column of $\operatorname{adj}\left(\hat{\mathrm{A}}_{s}\right)$ and polynomial $\hat{p}_{\mathrm{A}}^{+}$. Is is easily seen that the matrix constructed using the $j$ th column of $\operatorname{adj}\left(\hat{\mathrm{A}}_{s}\right)$ and polynomial $\tilde{\iota}\left(\hat{p}_{\mathrm{A}}^{+}\right)$is $\iota(\hat{\mathscr{H}})$. Moreover, one can check that

$$
\tilde{\mathrm{h}}_{i}^{\tau}=\xi_{1}^{r_{i}+(m-1) q-\tau} \iota\left(\hat{\mathrm{h}_{i}^{\tau}}\right), \quad \tau=0, \ldots, v-1 ; i=1, \ldots, \tilde{\nu} .
$$

Now we are ready to formulate the computational test for the SL-condition.

THeorem 6.4. An operator $(A, B)$ satisfies the SL-condition if and only if

(i) $\operatorname{rank}\left(\left(\hat{\Phi}_{1}^{22}\right)_{w}\right)=\tilde{v}-v$;

(ii) $\operatorname{rank}(\hat{\mathcal{H}})=\operatorname{rank}\left(\hat{\mathscr{H}}, \hat{k}^{l}\right)=v, l=1, \ldots, v$, where $\hat{k}^{1}, \ldots, \hat{k}^{v}$ is a basis of the vector space $\operatorname{ker}\left(\left(\hat{\Phi}_{1}^{22}\right)_{w}\right)$.

Note that elements of $\left(\hat{\Phi}_{1}^{22}\right)_{w}$ are in $\mathbb{K}$ because $\Phi_{1}^{22}$ is an operator on the boundary only. Hence $\operatorname{ker}\left(\left(\hat{\Phi}_{1}^{22}\right)_{w}\right)$ is really a vector space and not just a module.

Proof. From Theorem 6.3, we deduce that the SL-condition is equivalent to the following conditions: for all $\xi_{1} \neq 0$,

(1) $\operatorname{dim}\left(\operatorname{ker}\left(\left(\Phi_{1}^{22}\right)_{w}\right)\right)=v$, and

(2) $\operatorname{rank}(\mathcal{H}, d)=v$ for all $d \in \operatorname{ker}\left(\left(\Phi_{1}^{22}\right)_{w}\right)$.

First note that condition (1) is equivalent to saying: for all $\xi_{1} \neq 0, \operatorname{rank}\left(\left(\Phi_{1}^{22}\right)_{w}\right)=$ $\tilde{v}-v$. Then for a fixed $\xi_{1} \neq 0$, the matrix $\left(\Phi_{1}^{22}\right)_{w}$ is obtained from the matrix $\left(\hat{\Phi}_{1}^{22}\right)_{w}$ by multiplication of each $j$ th column by $\xi_{1}^{r_{j}}$ and each $i$ th row by $\xi_{1}^{\delta_{i}}$. Since the rank does not change under multiplication of columns and rows by nonzero elements, condition (1) is also equivalent to condition (i) of the theorem. 
Then, fixing some $\xi_{1} \neq 0$, we see that $d \in \operatorname{ker}\left(\left(\Phi_{1}^{22}\right)_{w}\right)$ has the following form:

$$
d=\left(\hat{d}_{1} \xi_{1}^{\tilde{m}+r_{1}}, \ldots, \hat{d}_{\tilde{\nu}} \xi_{1}^{\tilde{m}+r_{\tilde{v}}}\right)
$$

where $\hat{d}=\left(\hat{d}_{1}, \ldots, \hat{d}_{\tilde{v}}\right) \in \operatorname{ker}\left(\left(\hat{\Phi}_{1}^{22}\right)_{w}\right)$ and $\tilde{m} \in \mathbb{Z}$. Conversely, if $\hat{d} \in \operatorname{ker}\left(\left(\hat{\Phi}_{1}^{22}\right)_{w}\right)$, then $d \in \operatorname{ker}\left(\left(\Phi_{1}^{22}\right)_{w}\right)$ for any $\tilde{m}$ and $\xi_{1} \neq 0$.

Now (6.8), (6.10) and (6.9) imply that condition (2) is equivalent to the following statements.

$(2)^{\prime} \quad$ For all $\xi_{1} \neq 0, \operatorname{rank}(\tilde{\mathscr{H}}, d)=v$ for any $d \in \operatorname{ker}\left(\left(\Phi_{1}^{22}\right)_{w}\right)$.

$(2)^{\prime \prime} \operatorname{rank}(\iota(\hat{\mathscr{H}}), \hat{d})=v$ for any $\hat{d} \in \operatorname{ker}\left(\left(\hat{\Phi}_{1}^{22}\right)_{w}\right)$.

The equalities $\hat{d}=\iota(\hat{d})$ and $\operatorname{det}(\iota(C))=\iota(\operatorname{det}(C))$ for any matrix $C$, and the injectivity of $\iota$ yield that condition $(2)^{\prime \prime}$ is equivalent to $\operatorname{rank}(\hat{\mathscr{H}}, \hat{d})=v$ for any $\hat{d} \in \operatorname{ker}\left(\left(\hat{\Phi}_{1}^{22}\right)_{w}\right)$. But by elementary algebra this condition is equivalent to

$$
\operatorname{rank}(\hat{\mathscr{H}})=\operatorname{rank}\left(\hat{\mathscr{H}}, \hat{k}^{l}\right)=v, \quad l=1, \ldots, v,
$$

where $\hat{k}^{1}, \ldots, \hat{k}^{v}$ is a basis of $\operatorname{ker}\left(\left(\hat{\Phi}_{1}^{22}\right)_{w}\right)$.

Corollary 6.1. Let $v=\tilde{v}$. An operator $(A, B)$ satisfies the SL-condition if and only if $\operatorname{rank}(\hat{\mathscr{H}})=v$.

EXAmple 6.1. Consider the transformation of the two-dimensional Laplace equation $u_{20}+u_{02}=0$ to the first-order elliptic system

$$
A:\left\{\begin{array}{l}
y_{10}^{1}+y_{01}^{2}=0, \\
y_{01}^{1}-y_{10}^{2}=0, \\
y_{10}^{3}-y^{1}=0, \\
y_{01}^{3}-y^{2}=0,
\end{array} \quad \text { in } \mathbb{R}_{+}^{2}=\left\{x \in \mathbb{R}^{2}: x_{2}>0\right\}\right.
$$

with the following boundary conditions:

$$
B:\left\{\begin{array}{l}
y^{1}=0, \\
y^{3}=0,
\end{array} \quad \text { on } \partial \mathbb{R}_{+}^{2} .\right.
$$

Note that these boundary conditions are the Dirichlet condition $\left(y^{3}=0\right.$ on $\left.\partial \mathbb{R}_{+}^{2}\right)$ for the Laplace equation, and a differential consequence of the relations $y^{3}=0$ and $y_{10}^{3}-y^{1}=0$ on $\partial \mathbb{R}_{+}^{2}$ (the tangent part of the operator $A$ ). We will prove that these boundary conditions satisfy the SL-condition.

Now we will construct the component $\Phi_{1}^{22}$ of a compatibility operator $\Phi_{1}$ for the boundary value problem operator $(A, B)$. Note that the operator $(A, B)$ is normalised, and the tangent part of the operator $A$ is $A^{\tau} y=y_{10}^{3}-y^{1}$. Let us define a differential operator

$$
\Phi^{\tau} y=\left(A^{\tau} y, B y\right)=\left(y_{10}^{3}-y^{1}, y^{1}, y^{3}\right) \text {. }
$$

To construct a compatibility operator $\Phi_{1}^{\tau}$ for the operator $\Phi^{\tau}$, we compute the syzygy module for the module generated by the rows of the matrix

$$
\left(\begin{array}{ccc}
-1 & 0 & \xi_{1} \\
1 & 0 & 0 \\
0 & 0 & 1
\end{array}\right)
$$


Using Singular we get $\left(-1,-1, \xi_{1}\right)$, and hence

$$
\Upsilon^{\tau}\left(f^{\tau}, g^{1}, g^{2}\right)=-f^{\tau}-g^{1}+\frac{\partial g^{2}}{\partial x_{1}} .
$$

So the component $\Phi_{1}^{22}$ of the compatibility operator $\Phi_{1}$ and its weighted principal symbol with weights $r_{1}=r_{2}=0$ and $\delta_{1}=1$ are

$$
\Phi_{1}^{22}\left(g^{1}, g^{2}\right)=-g^{1}+\frac{\partial g^{2}}{\partial x_{1}}, \quad\left(\Phi_{1}^{22}\right)_{w}=\left(\begin{array}{ll}
0 & \xi_{1}
\end{array}\right), \quad\left(\hat{\Phi}_{1}^{22}\right)_{w}=\left(\begin{array}{ll}
0 & 1
\end{array}\right) .
$$

Let us consider the principal symbol of the operator $A$ :

$$
A=\left(\begin{array}{ccc}
\xi_{1} & \zeta & 0 \\
\zeta & -\xi_{1} & 0 \\
0 & 0 & \xi_{1} \\
0 & 0 & \zeta
\end{array}\right)
$$

So the characteristic polynomial of the operator $A$ is

$$
p_{\mathrm{A}}=\operatorname{gcd}\left(-\zeta^{3}-\zeta \xi_{1}^{2},-\zeta^{2} \xi_{1}-\xi_{1}^{3}\right)=\zeta^{2}+\xi_{1}^{2}, \quad \hat{p}_{\mathrm{A}}=\zeta^{2}+1, \quad \hat{p}_{\mathrm{A}}^{+}=\zeta-i .
$$

Thus $\operatorname{rank}\left(\left(\hat{\Phi}_{1}^{22}\right)_{w}\right)=\tilde{v}-v=1$. Hence condition (i) of Theorem 6.4 holds. Let us then consider the following submatrix of the dehomogenised principal symbol $\hat{A}$ :

$$
\hat{\mathrm{A}}_{123}=\left(\begin{array}{ccc}
1 & \zeta & 0 \\
\zeta & -1 & 0 \\
0 & 0 & 1
\end{array}\right)
$$

Computing with SINGULAR the adjoint of $\hat{\mathrm{A}}_{123}$, we get

$$
\operatorname{adj}\left(\hat{\mathrm{A}}_{123}\right)=\left(\begin{array}{ccc}
-1 & -\zeta & 0 \\
-\zeta & 1 & 0 \\
0 & 0 & -\zeta^{2}-1
\end{array}\right)
$$

Let $\hat{v}$ be the first column of $\operatorname{adj}\left(\hat{\mathrm{A}}_{123}\right)$. The vector $\hat{v}$ is nonzero modulo $\hat{p}_{\mathrm{A}}^{+}$, since the reduction of $\hat{v}$ with respect to $\hat{p}_{\mathrm{A}}^{+}$is $(-1,-i, 0)$. Note that

$$
\hat{\mathrm{B}}=\left(\begin{array}{lll}
1 & 0 & 0 \\
0 & 0 & 1
\end{array}\right) \text {. }
$$

So we get $\hat{h}=\hat{\mathrm{B}} \hat{v}=(-1,0)$. Reducing the elements of $\hat{h}$ with respect to $\hat{p}_{\mathrm{A}}^{+}$, we get $\hat{\mathrm{h}}=(-1,0)$. Since $v=1$, we have $\hat{\mathscr{H}}=\hat{\mathrm{h}}$. So $\operatorname{rank}(\hat{\mathscr{H}})=\operatorname{rank}(\hat{\mathscr{H}}, \hat{k})=1$, where $\hat{k}=(1,0)$ is a basis of the vector space $\operatorname{ker}\left(\left(\hat{\Phi}_{1}^{22}\right)_{w}\right)$. Hence condition (ii) of Theorem 6.4 holds, and so the boundary conditions (6.12) satisfy the SL-condition.

EXAMPLE 6.2. Let us consider the boundary value problem $\left(A_{0}, B\right)$ defined by $(5.13)$ and (5.14), where $A_{0}$ is the involutive form of the Stokes system.

The dehomogenised principal symbol of $A_{0}$ is

$$
\hat{\mathrm{A}}_{0}=\left(\begin{array}{ccc}
-\zeta^{2}-1 & 0 & 0 \\
0 & -\zeta^{2}-1 & 0 \\
0 & 0 & -\zeta^{2}-1 \\
0 & 0 & 0 \\
1 & \zeta & 0 \\
\zeta & \zeta^{2} & 0
\end{array}\right)
$$


Hence $\hat{p}_{\mathrm{A}}=\left(\zeta^{2}+1\right)^{2}$ and $v=2$. The part $\Phi_{1}^{22}$ of the compatibility operator for $\left(A_{0}, B\right)$ was computed in (5.15). The dehomogenised weighted symbol of $\Phi_{1}^{22}$ is

$$
\left(\hat{\Phi}_{1}^{22}\right)_{w}=\left(\begin{array}{ccccc}
1 & -2 & 1 & 3 & 0 \\
0 & 0 & 0 & 0 & 0 \\
0 & 0 & 0 & 0 & 0 \\
0 & 0 & 0 & 0 & 0 \\
0 & 0 & 0 & 0 & 0 \\
0 & 0 & 0 & 0 & 1
\end{array}\right)
$$

So $\operatorname{rank}\left(\left(\hat{\Phi}_{1}^{22}\right)_{w}\right)=2 \neq \tilde{v}-v=3$. Hence condition (i) of Theorem 6.4 does not hold, and $\left(A_{0}, B\right)$ does not satisfy the SL-condition.

\section{Conclusions and perspectives}

We have shown above how to check constructively the validity of the SL-condition in the two-variable case. This case is already important in PDE computations; however, it would clearly be desirable to extend our results to the case of arbitrary numbers of variables.

When there are 'too many' boundary conditions, we need the compatibility operator to perform the test. One may wonder why one should try to impose more boundary conditions than are strictly necessary. On the other hand, one could ask the same question about PDE systems; yet it is clear that analysing only square systems is not enough. Perhaps the same will happen with boundary conditions: when the systems get more complicated, situations may arise where it is 'natural' or important to consider 'too many' boundary conditions. However, as far as we know, there has been no work in this direction in the numerical analysis of PDEs, so it is at present not clear how important this will be in the future.

In formulating our results we have supposed that the system is given in a specific (local) coordinate system. Obviously, in practice we do not want to make explicit coordinate transformations, so a natural goal would be to formulate the test in the original coordinate system. On a more technical level, it is not clear whether it is really necessary to factor the characteristic polynomial. Explicit factorisation of even quite simple polynomials can be very time-consuming. Moreover, if we can avoid factoring in the two-variable case, perhaps this alternative approach could be generalised to the many-variable case. Finally, a natural way to extend our work is to apply this approach also to overdetermined parabolic and hyperbolic systems. We hope to address all these issues in future papers.

Acknowledgement. The first author gratefully acknowledges the financial support of the Academy of Finland, Grant 108394.

\section{Appendix A. Useful commands in computer algebra systems SINGULAR and MUPAD}

As we have seen, symbols of differential operators are naturally viewed as modules generated by the rows of the symbol matrix. However, in Singular (and in commutative algebra textbooks in general) modules are generated by the columns of matrices. Hence in the following commands we will often need to transpose matrices. To use some of the commands in SINGULAR one needs to load some appropriate libraries. To access all libraries one can use the following command.

$>$ LIB "all.lib"; 
Appendix A.1. Finding the splitting field of the characteristic polynomial and its roots

$>$ ring $r=0, y, d p$

$>$ poly $p=y^{6}+y^{4}+y^{2}+1$

$>$ def $r 1=\operatorname{Roots}(p)$

$>$ setring $r 1$;

$>$ roots;

$a^{3},-a^{3}, a,-a,-a^{2}, a^{2}$

$>$ minpoly;

$a^{4}+1$

Appendix A.2. Determining the admissible combinations of the roots of the characteristic polynomial

$>$ ring $r 2=$ complex $, a, d p$;

$>$ poly $\operatorname{pmin}=a^{4}+1$;

$>$ list $l=\operatorname{solve}($ pmin $)$;

$>$ poly $\operatorname{root} 1=a^{3}$;

$>$ substitute $($ root $1, a, l[1])$;

$-0.70710678+i 0.70710678$

Appendix A.3. Computations in the SL-test

$>\operatorname{ring} r=(0, a), z, d p ;$ minpoly $=a^{2}+1$;

$>$ poly $p=z-a$;

$>$ matrix $\operatorname{ma}[3][3]=1, z, 0, z,-1,0,0,0,1$;

$>$ matrix $m b[2][3]=1,0,0,0,0,1$;

// Finding of a vector $v$

$>$ matrix $\operatorname{maadj}=\operatorname{adjoint}(m a)$;

$>$ ideal $i d=p$; ideal $i d s t d=\operatorname{std}(i d)$;

$>$ int $m=\operatorname{ncols}(\operatorname{maad} j)$;

int $i$; int $j$;

for $(j=1 ; j<=m ; j=j+1)$

\{

for $(i=1 ; i<=m ; i=i+1)$

\{

$\operatorname{maad} j[i, j]=\operatorname{reduce}(\operatorname{maad} j[i, j], i d s t d)$;

\}

\}

// After the reduction we notice that the first column is nonzero.

$>$ matrix $v[3][1]=-1,-z, 0$;

// Definition of a vector $h$ and construction of a vector $h$

$>$ matrix $m h=m b * v$;

$>$ int $m=\operatorname{ncols}(m h)$;

int $n=\operatorname{nrows}(m h)$;

int $i$; int $j$;

for $(j=1 ; j<=m ; j=j+1)$ 


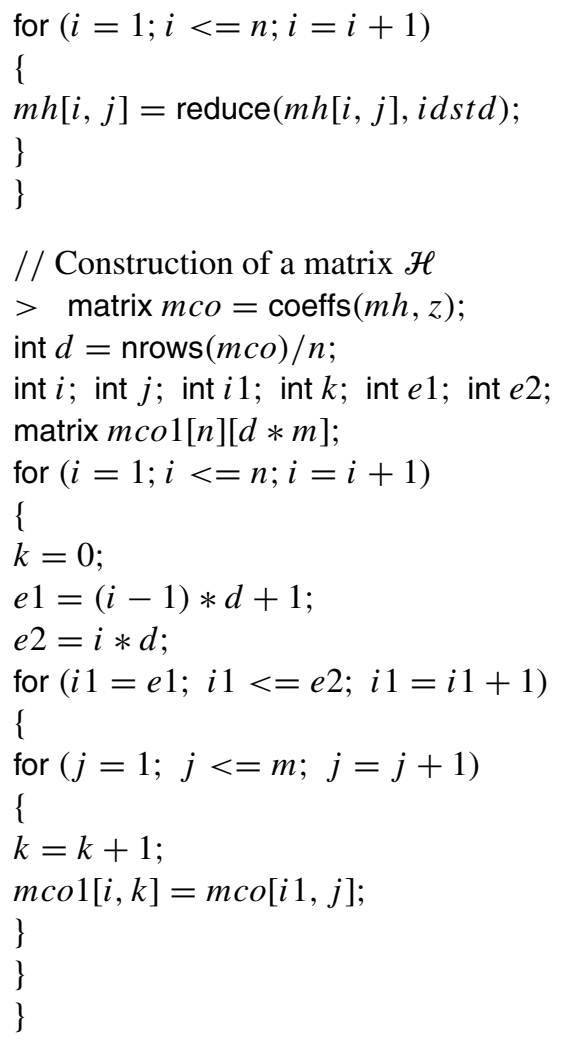

Appendix A.4. Computation of the compatibility operator when there is no boundary operator

$>$ ring $r=0,(z, x), d p$;

$>$ matrix $m[3][3]=-1,0, x, 1,0,0,0,0,1$;

$>$ matrix $s=\operatorname{transpose}(\operatorname{syz}(\operatorname{transpose}(m)))$;

Appendix A.5. Computation of the tangent part of the operator A

$>$ ring $r=0,(z, x),(l p, c)$;

// Here we define TOP ordering with $z>x$.

$>$ module $m=[0,0,0,-x, 0,1,-z, 0],[0,0,0, z,-x, 0,0,1]$, $[0,0,0,0,0,-x, 0,-z],[x, 0,0,-1,0,0,0,0],[z, 0,0,0,0,0,-1,0]$, $[0, x, 0,0,-1,0,0,0],[0, z, 0,1,0,0,0,0],[0,0, x, 0,0,-1,0,0]$, $[0,0, z, 0,0,0,0,-1],[0,0,0, z, 0,0,-x, 0],[0,0,0, x, z, 0,0,0]$, $[0,0,0,0,0, z, 0,-x]$;

$>$ module $m s=\operatorname{transpose}(\operatorname{std}(m))$;

$>\operatorname{print}(m s)$;

$0,0,0,0, x, 0,-x,-1$,

$0,0, x, 0,0,-1,0,0$,

$0, x, 0,0,-1,0,0,0$,

$x, 0,0,-1,0,0,0,0$,

$0,0,0,0,0, x, 0, z$, 

$0,0,0, x, 0,-1, z, 0$,
$0,0,0,0,0, z, 0,-x$,
$0,0,0, x, z, 0,0,0$,
$0,0,0, z, 0,0,-x, 0$,
$0,0, z, 0,0,0,0,-1$,
$0, z, 0,1,0,0,0,0$,
$z, 0,0,0,0,0,-1,0$

Appendix A.6. Completion of a system to involutive form with MUPAD

$$
\begin{aligned}
&> \text { LDF }:=\text { Dom::LinearDifferentialFunction(Vars }=[[x 1, x 2, x 3],[y]], \\
& \text { Rest }=[\text { Types }=\text { "Indep"] }): \\
&> \text { sys : }=\operatorname{map}([y([x 3, x 3])-x 2 * y([x 1, x 1]), y([x 2, x 2])], \text { LDF }): \\
&>\text { detools }:: \text { complete }(s y s, \text { Output }=3) ;
\end{aligned}
$$

\section{References}

1. W. Adams and P. Loustaunau, An introduction to Gröbner bases, Graduate Studies in Mathematics 3 (Amer. Math. Soc., Providence, RI, 1994). 289, 296

2. S. Agmon, A. Douglis and L. Nirenberg, 'Estimates near the boundary for solutions of elliptic partial differential equations satisfying general boundary conditions. I', Comm. Pure Appl. Math. 12 (1959) 623-727. 287, 290, 301

3. S. Agmon, A. Douglis and L. Nirenberg, 'Estimates near the boundary for solutions of elliptic partial differential equations satisfying general boundary conditions. II', Comm. Pure Appl. Math. 17 (1964) 35-92. 287, 300, 301, 304

4. M. S. Agranovich, 'Elliptic boundary problems', Partial differential equations IX, ed. M. S. Agranovich, Yu. V. Egorov and M. A. Shubin, Encyclopaedia of Mathematical Sciences 79 (Springer, Berlin/Heidelberg, 1997) 1-144. 287, 298, 301

5. J. R. BASTIDA, Field extensions and Galois theory, Encyclopaedia of Mathematics and its Applications 22 (Addison-Wesley Publishing Company, 1984). 288, 290

6. J. Belanger, M. Hausdorf and W. Seiler, 'A MuPAD library for differential equations', Computer Algebra in Scientific Computing - CASC 2001 (ed. V. G. Ghanza, E. W. Mayr and E. V. Vorozhtsov; Springer, Berlin/Heidelberg, 2001) 25-42. 295

7. A. Douglis and L. Nirenberg, 'Interior estimates for elliptic systems of partial differential equations', Comm. Pure Appl. Math. 8 (1955) 503-538. 298

8. P. I. Dudnikov and S. N. SAMborski, 'Noetherian boundary value problems for overdetermined systems of partial differential equations', Dokl. Akad. Nauk SSSR 258 (1981) 283-288 (in Russian), Soviet Math. Dokl. 23 (1981) (English translation). $287,295,310$

9. P. I. Dudnikov and S. N. SAmborski, 'Linear overdetermined systems of partial differential equations. Initial and initial-boundary value problems', Partial differential equations VIII, ed. M. A. Shubin, Encyclopaedia of Mathematical Sciences 65 (Springer, Berlin/Heidelberg, 1996) 1-86. 287, 293, 318

10. D. EISENBUd, Commutative algebra with a view toward algebraic geometry, Graduate Texts in Mathematics 150 (Springer, New York, 1995). 288, 289 
11. J. Gerhard, W. Oevel, F. Postel and S. Wehmeier, MuPAD tutorial (Springer, 2000), http: / /www. mupad.de/. 288, 295

12. G.-M. Greuel and G. Pfister, A Singular introduction to commutative algebra (Springer, 2002). 288, 290

13. G.-M. Greuel, G. Pfister and H. Schönemann, 'Singular 2.0. a computer algebra system for polynomial computations', http://www.singular.uni-kl.de. 288, 306

14. E. L. InCE, Ordinary differential equations (Dover, 1956). 290

15. K. Krupchy , W. SeIler and J. TuOmela, 'Overdetermined elliptic PDEs', J. Found. Comp. Math., to appear, 288, 298, 308

16. YA. B. LopatinsKiJ, 'A method of reduction of boundary value problems for systems of differential equations of elliptic type to a system of regular integral equations', $U k r$. Mat. Zh. 5 (1953) 123-151. 287

17. B. Mohammadi and J. TuOmela, 'Simplifying numerical solution of constrained PDE systems through involutive completion', M2AN Math. Model. Numer. Anal., to appear. 287

18. T. MorA, Solving polynomial equation system I: the Kronecker-Duval philosophy, Encyclopaedia of Mathematics and its Applications 88 (Cambridge University Press, 2003). 288

19. J. F. Pommaret, Systems of partial differential equations and Lie pseudogroups, Mathematics and its Applications 14 (Gordon and Breach Science Publishers, 1978). 292, 293, 294, 295, 310, 312

20. J. F. Pommaret and A. Quadrat, 'Algebraic analysis of linear multidimensional control systems', IMA J. Math. Control Inform. 16 (1999) 275-297. 298

21. D. SAunders, The geometry of jet bundles, London Math. Soc. Lecture Note Series 142 (Cambridge University Press, 1989). 292

22. W. M. SEILER, 'Involution - the formal theory of differential equations and its applications in computer algebra and numerical analysis', Habilitation thesis, Dept. of Mathematics, University of Mannheim, 2001 (Springer, to appear). 294, 295, 312

23. Z. YA. Shapiro, 'On general boundary problems for equations of elliptic type', Izvestiya Akad. Nauk SSSR. Ser. Mat 17 (1953) 539-562 (in Russian). 287

24. D. SPEnCER, 'Overdetermined systems of linear partial differential equations', Bull. Amer. Math. Soc 75 (1969) 179-239. 294, 295

25. N. N. TARKhanov, translated from the 1990 Russian original by P. M. Gauthier and revised by the author, Complexes of differential operators, Mathematics and its Applications 340 (Kluwer, 1995). 293, 309, 310, 311

Katsiaryna Krupchyk krupchyk@joyx.joensuu.fi

http://www.joensuu.fi/mathematics/department/personnel/krupchyk.htm Jukka Tuomela jukka.tuomela@joensuu.fi

http://www.joensuu.fi/mathematics/department/personnel/tuomela.htm

Dept. of Mathematics

University of Joensuu

Finland 